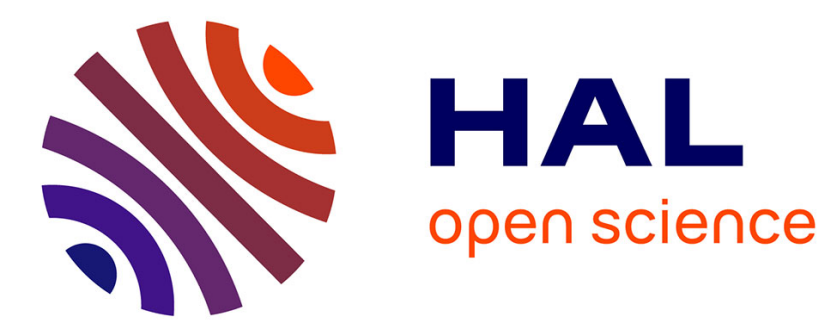

\title{
Micromechanical modeling of hardening mechanisms in commercially pure alpha-titanium in tensile condition
}

\author{
Kékéli Eva K. Amouzou, Thiebaud Richeton, Amandine Roth, Mikhail
}

Lebyodkin, Tatiana Lebedkina

\section{- To cite this version:}

Kékéli Eva K. Amouzou, Thiebaud Richeton, Amandine Roth, Mikhail Lebyodkin, Tatiana Lebedkina. Micromechanical modeling of hardening mechanisms in commercially pure alpha-titanium in tensile condition. International Journal of Plasticity, 2016, 80, pp.222-240. 10.1016/j.ijplas.2015.09.008 . hal-01514731

\author{
HAL Id: hal-01514731 \\ https://hal.univ-lorraine.fr/hal-01514731
}

Submitted on 17 Dec 2019

HAL is a multi-disciplinary open access archive for the deposit and dissemination of scientific research documents, whether they are published or not. The documents may come from teaching and research institutions in France or abroad, or from public or private research centers.
L'archive ouverte pluridisciplinaire HAL, est destinée au dépôt et à la diffusion de documents scientifiques de niveau recherche, publiés ou non, émanant des établissements d'enseignement et de recherche français ou étrangers, des laboratoires publics ou privés. 


\title{
Micromechanical modeling of hardening mechanisms in commercially pure $\alpha$-titanium in tensile condition
}

\author{
K.E.K. Amouzou ${ }^{\text {a }}$, T. Richeton ${ }^{\text {a, }}{ }^{*}$, A. Roth ${ }^{\text {b }}$, M.A. Lebyodkin ${ }^{\text {a }}$, T.A. Lebedkina ${ }^{a}$ \\ a Laboratoire d'Etude des Microstructures et de Mécanique des Matériaux (LEM3), UMR 7239, CNRS / Université de Lorraine, \\ Ile du Saulcy, 57045 Metz, France \\ ${ }^{\mathrm{b}}$ Asco Industrie - CREAS, Avenue de France, 57300 Hagondange, France
}

\section{A R T I C L E I N F O}

\section{Keywords:}

A. Microstructures

B. Anisotropic material

B. Crystal plasticity

B. Polycrystalline material

C. Mechanical testing

\begin{abstract}
A B S T R A C T
Tensile tests on commercially pure $\alpha$-titanium show a three-stage behavior giving rise to a well on the strain dependence of the work hardening. An opposite strain rate effect on the well depth is found whether specimens are elongated along the rolling or the transverse direction. Slip lines analysis reveals an initial predominance of prismatic slip, particularly pronounced in specimens strained along the rolling direction. The relative activity of prismatic slip is then observed to decrease with the samples deformation. These results provide grounds for elaboration of an elasto-viscoplastic self-consistent model based on the translated field method and an affine linearization of the viscoplastic flow rule, and capable of explaining such peculiar work hardening behavior. The model considers crystal plasticity and deals separately with mobile dislocation density and dislocation velocity. It assumes lower strain rate sensitivity as well as higher dislocation multiplication rate for prismatic systems. Based on these assumptions, the model reproduces correctly the stress -strain curves and gives sound estimates of Lankford coefficients, prismatic slip activity and textures evolution. Most importantly, the opposite effect of strain rate on the well depth with regard to the orientation of the tensile axis is qualitatively retrieved, which allows putting forward an explanation of the observed phenomena.
\end{abstract}

\section{Introduction}

At ambient temperature, Ti has a hexagonal close-packed (hcp) structure which is characterized by a high anisotropy of glide resistance. There is a general agreement that prismatic slip is the easiest deformation mode (Churchman, 1954; Philippe et al., 1995; Zaefferer, 2003; Salem et al., 2005; Wu et al., 2007; Gong and Wilkinson, 2009; Gurao et al., 2011; Li et al., 2013; Warwick et al., 2012; Benmhenni et al., 2013; Gloagen et al., 2013; Barkia et al., 2015). However, less consensus has been found concerning the ranking of other possible slip and twinning systems, especially the corresponding values of the critical resolved shear stress (CRSS) which closely depend on material composition. Their knowledge is however of primary importance for a coherent choice of the parameters used in plasticity models. In particular, glide of $\langle c+a>$ dislocations or twinning modes must be involved in the deformation of a Ti crystal so that it could support arbitrary shape changes. Hence, twinning can occur in titanium as observed in many experiments (e.g., Monteiro and Reed-

\footnotetext{
* Corresponding author. Tel.: +33 3873153 62; fax: +33 387315366 .

E-mail address: thiebaud.richeton@univ-lorraine.fr (T. Richeton).
} 
Hill (1973); Nemat-Nasser et al. (1999); Salem et al. (2002, 2003, 2006); Nixon et al. (2010); Wang et al. (2010); Ghaderi and Barnett (2011); Gurao et al. (2011); Wang et al. (2012); Li et al. (2013); Becker and Pantleon (2013); Roth et al. (2014); Barkia et al. (2015)). On the other hand, Ti can also deform with a very negligible twin volume fraction (e.g., Nixon et al. (2010); Ghaderi and Barnett (2011); Roth et al. (2014)). The proneness to twinning manifestly depends on material (composition (Monteiro and Reed-Hill, 1973; Li et al., 2014; Barkia et al., 2015), grain size (Ghaderi and Barnett, 2011; Wang et al., 2012), texture (Nixon et al., 2010; Wang et al., 2010; Li et al., 2014)) and loading conditions (temperature (Monteiro and Reed-Hill, 1973; Li et al., 2013, 2014), strain rate (Nemat-Nasser et al., 1999; Gurao et al., 2011), loading mode (Salem et al., 2003; Nixon et al., 2010)).

In particular, twinning is often evoked to explain the strain hardening behavior of Ti (e.g., Salem et al. (2006); Knezevic et al. (2013)). In compression condition, three-stage behavior is notably reported. The initial stage A is characterized by a decreasing strain hardening rate $\Theta=d \Sigma / d E^{p}$ (where $\Sigma$ designates the macroscopic stress and $E^{p}$ the macroscopic plastic strain). It is followed by an increase in $\Theta$ during stage B and, finally, a new decrease in stage C. Salem et al. (2002, 2003, 2006) found that the onset of twinning correlates with stage B. Accordingly, they proposed to ascribe the increase in the strain hardening to the effect of twin boundaries on the resistance to dislocation glide, and the decrease in stage $C$ to saturation of the twin volume fraction.

However, recent experiments from Roth et al. (2014) showed that the three-stage character of Ti strain hardening behavior also manifests itself in conditions that induce almost no twinning (twin volume fraction $<0.5 \%$ ), such as tensile loading of commercially pure (CP) $\alpha$-Ti with $9 \mu \mathrm{m}$ grain size along the rolling direction. Tests on differently oriented samples showed an ambiguous role of twins in tensile condition. On the one hand, for samples stretched along the transverse direction, these experiments revealed a more significant twin volume fraction (3 to 6\%) and a more pronounced tendency to three-stage behavior, which was weaker than in compression tests generally characterized by twin volume fraction an order of magnitude higher (Nemat-Nasser et al., 1999; Salem et al., 2002, 2003, 2006). At the same time, the work hardening rate was considerably stronger all over the deformation curve for samples deformed along the rolling direction, i.e., those with the lowest twin volume fraction. The latter observation is in contradiction with the hardening effect of twins, resulting from the dynamic Hall-Petch effect and/or Basinski effect (Basinski et al., 1997). Such effects could have naturally been conjectured to explain the increase of $\Theta$ during stage B of tensile curves, similar to the interpretation applied in the case of compression. Another important observation concerned the effect of the imposed strain rate on the three-stage behavior, which was found to be opposite for the two kinds of elongation directions: the trend to formation of the low hardening stage A was the strongest at the lowest/highest strain rate for samples deformed along the rolling/transverse direction, respectively (Roth et al., 2014). This inversion of the sign of the strain rate effect neither can be explained by the supposed effects of twins. Given the very low twin volume fraction recorded, it was thus conjectured, by Roth et al. (2014), that twins played a secondary role in the tensile deformation of the tested Ti samples. It is noteworthy that such hypothesis is corroborated by some literature results. In the tensile experiments reported by Ghaderi and Barnett (2011), yield point elongation was actually more pronounced for the finest grain size samples, i.e., the ones where twinning was insignificant, and Becker and Pantleon (2013) observed a weaker trend to three stage behavior in tensile condition compared to Roth et al. (2014), in spite of much more intense twinning.

Hence, the data in tensile condition do not support the conjecture of twinning as the mechanism controlling the threestage strain hardening behavior. Another mechanism could be dynamic strain aging of dislocations caused by their interactions with solute atoms, as suggested by Nemat-Nasser et al. (1999) to explain compression behavior of CP Ti. Nevertheless, this explanation does not apply to ambient temperature because the dynamic strain aging is only essential in $\mathrm{Ti}$ in the temperature range of 500-850 K, as shown by Doner and Conrad (1973); Reed-Hill et al. (1995); Lecomte et al. (1997) and discussed by Salem et al. (2002, 2003, 2006). Alternatively, static solute aging of dislocations could account for the initial low work hardening rate during stage $A$ as the occurrence of the Piobert-Lüders phenomenon. However, no propagation of a deformation band was observed by Roth et al. (2014) from high-temporal resolution extensometry performed with the aid of a CCD camera. It was observed instead that the plastic flow started simultaneously in the whole specimen and displayed a high degree of homogeneity, thus invalidating a priori this surmise. As a consequence, it is necessary to examine the three-stage hardening character of tensile deformation of $\mathrm{CP}$ Ti from the viewpoint of mechanisms based on dislocations glide only. For instance, Conrad (1981) and Naka (1983) both suggested ascribing the yield plateau observed in $\mathrm{Ti}$ to fast multiplication of mobile dislocations. In order to explain qualitatively the strain rate effect on the proneness to three-stage behavior, Roth et al. (2014) suggested to take into account that the strain rate sensitivity of stress (SRSS) may be different for different slip families. However, without considering the dislocation multiplication effects, this suggestion led to an unrealistic assumption of a stronger SRSS for prismatic glide, as compared with pyramidal glide.

The objective of the present paper is to propose a model, based on dislocations glide mechanisms, that can account for the work hardening evolution in tensile condition. First, dislocation slip system activity is investigated experimentally, with the objective to provide missing criteria for selection of the relevant mechanisms. Interrupted tensile tests and slip lines characterization in the microscope are used for this task (Sections 2 and 3). Then, an elasto-viscoplastic self-consistent polycrystalline model that integrates mobile dislocation multiplication and different strain rate sensitivities is developed (Section 4). The capacity of the polycrystalline model to reproduce the experimental observations, including stress-strain curves, work hardening behavior, relative slip system activities, Lankford coefficients and textures evolution are analyzed in Section 4. Discussion and conclusions follow. 
Table 1

Chemical composition of commercially pure titanium T40.

\begin{tabular}{llllll}
\hline Elements & $\mathrm{H}$ & $\mathrm{C}$ & $\mathrm{N}$ & $\mathrm{O}$ & $\mathrm{Ti}$ \\
\hline Composition ppm (wt.) & 3 & 52 & 41 & 1062 & \\
\hline
\end{tabular}

\section{Experimental technique}

The experiments were carried out with samples machined from the same CP Ti sheet as Roth et al. (2014). The material composition is recalled in Table 1 . The initial microstructure was fully recrystallized without any twin, and the grains were equiaxed with an average size around $9 \mu \mathrm{m}$. As can be seen in Fig. 1, the initial texture was typical of rolled titanium (Wang and Huang, 2003; Benmhenni et al., 2013; Becker and Pantleon, 2013; Roth et al., 2014), with basal planes tilted $30^{\circ} \pm 10^{\circ}$ from the normal toward the transverse direction. The samples with a gage section of $30 \times 7 \times 1.62 \mathrm{~mm}^{3}$ were cut parallel to either the rolling direction (hereinafter referred to as RD-samples) or the transverse direction (TD-samples). The specimens were deformed at room temperature at a constant crosshead velocity corresponding to initial applied strain rates of $5 \cdot 10^{-4} \mathrm{~s}^{-1}$ and $8 \cdot 10^{-3} \mathrm{~s}^{-1}$. A sensor arm extensometer was used to measure accurately the elongation of the specimen gage lengths. In order to estimate twin volume fractions and characterize slip systems activity, tensile tests were interrupted firstly around stage B ( $0.5 \%$ of plastic strain for RD-samples and $\sim 0.8 \%$ for TD-samples) and then at necking. Some tests were interrupted at large enough strain before necking ( $\sim 9.0 \%$ of plastic strain for RD-samples and $\sim 6.2 \%$ for TD-samples) in order to measure Lankford coefficients. Disregarding the sample volume change, the Lankford coefficient $r$, which is the ratio between the in-plane and the out-of-plane transverse strains, was estimated as

$$
r=\frac{E_{22}^{p}}{E_{33}^{p}}=-\frac{E_{22}^{p}}{E_{11}^{p}+E_{22}^{p}}
$$

where $E_{i j}^{p}$ is the macroscopic plastic strain tensor, 1 the tensile direction, 2 the in-plane transverse one and 3 the throughthickness one. Finally, some other tests were carried out until fracture. Each test was repeated at least two times.

In order to perform slip lines analysis, the tensile samples were mechanically polished before the tests with SiC paper down to \#4000 finish and then electrolytically polished for $30 \mathrm{~s}$ in a solution of $20 \%$ perchloric and $80 \%$ methanol $\left(25 \mathrm{~V}, 5^{\circ} \mathrm{C}\right)$. Then, for each sample strained until stage B or necking, between 10 and 15 images, including a total of 100-150 grains, were taken in a FEG-SEM (Field Emission Gun - Scanning Electron Microscope). After that, lattice orientations of the same areas were characterized by the EBSD (Electron Backscatter Diffraction) method on a JEOL JSM-6490 SEM. The orientation maps were constructed with $0.25 \mu \mathrm{m}$ step intervals using an AZtec (Oxford Instruments) EBSD system.

Slip planes were identified by comparison between the angle made by the slip trace at the sample surface with the loading axis, which is directly observed on the SEM image, and the one which can be computed from the EBSD Euler angles of the grain. Thirty slip systems were considered in the computations, which correspond to the 5 slip families that have been observed in Ti (Xiaoli et al., 1994): Prismatic $(P)$, Basal $(B)$, Pyramidal $\langle a\rangle\left(\Pi_{1}^{\langle a\rangle}\right)$, 1st order Pyramidal $\langle c+a\rangle\left(\Pi_{1}^{\langle c+a\rangle}\right)$ and 2nd order Pyramidal $\left(\Pi_{2}^{\langle c+a\rangle}\right.$ ) (see Table 2). From this method, no information can be obtained on the slip direction. Hence, it is not possible to distinguish between $\Pi_{1}^{\langle a\rangle}$ and $\Pi_{1}^{\langle c+a\rangle}$ families which share the same slip planes. Most of the time, the agreement between the observed and the computed angles was very good. However, due to some possible inaccuraccy in the method (sample misalignment, not perfectly straight slip line, inaccurate EBSD indexation), a slip family was identified when the angles difference was less than $5^{\circ}$. When multiple families were within the accuracy of $5^{\circ}$, the one with the computed angle
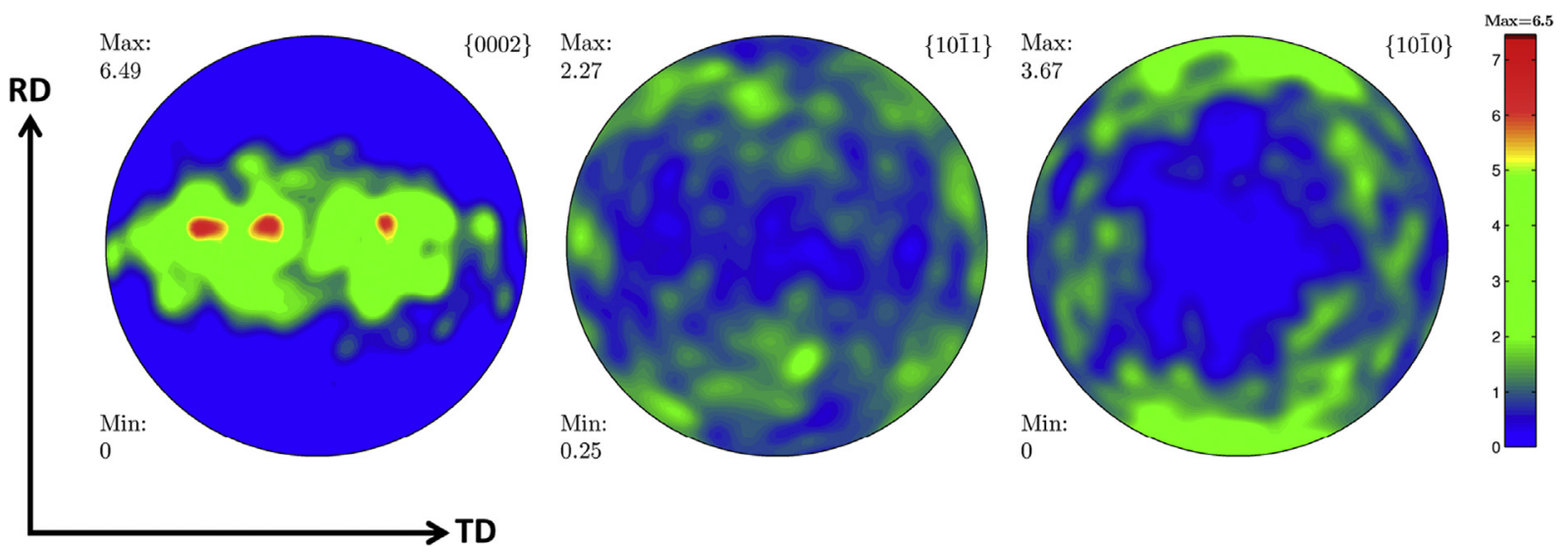

Fig. 1. Pole figures for an as-received sample measured by EBSD (7 08,771 orientations) and plotted with the MTEX toolbox (Bachmann et al., 2010). 
Table 2

Slip families observed in Ti.

\begin{tabular}{llc}
\hline Slip family & Indices & Number of systems \\
\hline$P$ & $\{1 \overline{1} 00\}[11 \overline{2} 0]$ & 3 \\
$B$ & $\{0001\}[1 \overline{1} 0]$ & 3 \\
$\Pi_{1}^{<a>}$ & $\{1 \overline{1} 01\}[11 \overline{2} 0]$ & 6 \\
$\Pi_{1}^{\langle c+a\rangle}$ & $\{1 \overline{1} 01\}[2 \overline{1} \overline{1} \overline{3}]$ & 12 \\
$\Pi_{2}^{\langle c+a\rangle}$ & $\{2 \overline{1} \overline{1} 2\}[2 \overline{1} \overline{1} \overline{3}]$ & 6 \\
\hline
\end{tabular}

Table 3

Quantitative estimates of twin volume fraction. T stands for $\{10 \overline{1} 2\}[\overline{1} 011]$ extension twins and C for $\{11 \overline{2} 2\}[11 \overline{2} \overline{3}]$ contraction twins.

\begin{tabular}{lllll}
\hline Orientation & Strain-rate & Stage B & Necking & Fracture \\
\hline TD & $5 \cdot 10^{-4} \mathrm{~s}^{-1}$ & $1.9 \% \mathrm{~T} ; 0 \% \mathrm{C}$ & $5.7 \% \mathrm{~T} ; 0 \% \mathrm{C}$ & - \\
& $8 \cdot 10^{-3} \mathrm{~s}^{-1}$ & $1.1 \% \mathrm{~T} ; 0 \% \mathrm{C}$ & - & $2.6 \% \mathrm{~T} ; 0 \% \mathrm{C}$ \\
$\mathrm{RD}$ & $5 \cdot 10^{-4} \mathrm{~s}^{-1}$ & $0.4 \% \mathrm{~T} ; 0.5 \% \mathrm{C}$ & $0 \% \mathrm{~T} ; 0 \% \mathrm{C}$ & - \\
& $8 \cdot 10^{-3} \mathrm{~s}^{-1}$ & $0 \% \mathrm{~T} ; 0 \% \mathrm{C}$ & - & $0.2 \% \mathrm{~T} ; 0.3 \% \mathrm{C}$ \\
\hline
\end{tabular}

Table 4

Frequency of observed slip families around stage B, as deduced from the procedure of slip line analysis described in Section 2.

\begin{tabular}{llllll}
\hline Orientation & Strain-rate & $P$ & $B$ & $\Pi_{1}^{<a>}+\Pi_{1}^{\langle c+a\rangle}$ & $23 \%$ \\
\\
TD & $5 \cdot 10^{-4} \mathrm{~s}^{-1}$ & $65 \%$ & $1 \%$ & $14 \%$ & $11 \%$ \\
& $8 \cdot 10^{-3} \mathrm{~s}^{-1}$ & $73 \%$ & $0 \%$ & $4 \%$ & $13 \%$ \\
$\mathrm{RD}$ & $5 \cdot 10^{-4} \mathrm{~s}^{-1}$ & $82 \%$ & $0 \%$ & $8 \%$ & $14 \%$ \\
& $8 \cdot 10^{-3} \mathrm{~s}^{-1}$ & $81 \%$ & $0 \%$ & $8 \%$ \\
\hline
\end{tabular}

the closest to the observed angle was chosen. If the two closest angles had less than $1^{\circ}$ discrepancy, the grain was excluded from the statistics. 94\% of the grains had only one observable slip system around stage B and $88 \%$ at necking. Hence, for simplicity, grains with 2 observable slip systems were also excluded from the statistics. Finally, frequency of observed slip families were computed from the total number of grains kept in the statistics.

Concerning twins, they were identified from the axis and the angle associated to the minimum misorientation with the parent phase within a tolerance of $\pm 5^{\circ}$. The twin volume fraction was then estimated by the intercept method (see details in the paper of Roth et al. (2014)). Finally, alongside with the EBSD analysis, optical microscopy was used to verify the accuracy of the estimated volume fraction over the whole sample (not shown), and hence validate the EBSD analysis.

\section{Slip and twinning characterization}

The above-described analysis reveals the occurrence of two kinds of twins: $\{10 \overline{1} 2\}[\overline{1} 011]$ extension twins and $\{11 \overline{2} 2\}[11 \overline{2} \overline{3}]$ contraction twins. Table 3 gives their respective twin volume fraction. The total twin volume fraction in RDspecimens remains tremendously small during all the tensile test. TD-samples show a greater amount of twins, with smaller fractions around stage B ( 1 to $2 \%$ ) than at the end of the test ( 3 to $6 \%$ ). The very small fractions measured around stage $\mathrm{B}$ thus confirm that twinning is unlikely to be the mechanism responsible for the observed strain hardening increase in stage B.

Other important observations follow from Tables 4 and 5 which provide, respectively, the frequency of slip families observed around stage $B$ and at necking. First, these results indicate that $P$ glide is more present in RD-samples than in TDsamples. This qualitative conclusion agrees with both the Schmid factor analysis and the lower yield stress reported for RDsamples (Roth et al., 2014; Won et al., 2015), the latter being related to the lower CRSS value for $P$ glide (Churchman, 1954; Philippe et al., 1995; Salem et al., 2005; Zaefferer, 2003; Wu et al., 2007; Gong and Wilkinson, 2009; Gurao et al., 2011; Li et al., 2013; Warwick et al., 2012; Benmhenni et al., 2013; Gloagen et al., 2013; Barkia et al., 2015). The proportion of pyramidal slip is also observed to increase in the course of the test for both orientations, which can thus be considered as a possible mechanism of $\Theta$ increase during stage B. Otherwise, Tables 4 and 5 allow rectifying some suggestions made by Roth et al. (2014). So, the present results show that the fraction of the grains initially deforming by pyramidal slip is not preponderant in either kind of samples, prismatic glide being essential even in TD-samples. Furthermore, the data contradict the

Table 5

Frequency of observed slip families at necking, as deduced from the procedure of slip line analysis described in Section 2.

\begin{tabular}{lllll}
\hline Orientation & Strain-rate & $P$ & $B$ & $\Pi_{1}^{<a>}+\Pi_{1}^{\langle c+a\rangle}$ \\
\hline TD & $5 \cdot 10^{-4} \mathrm{~s}^{-1}$ & $51 \%$ & $0 \%$ & $26 \%$ \\
RD & $5 \cdot 10^{-4} \mathrm{~s}^{-1}$ & $65 \%$ & $0 \%$ & $17 \%$ \\
\hline
\end{tabular}


assumption of a stronger SRSS for $P$ systems since their frequency is rather observed to increase with the applied strain rate (Table 4). Henceforth, it is suggested instead than the SRSS should be weaker for $P$ systems (see also measurements of activation volume by Levine (1966)).

It should be underlined that the results of Tables 4 and 5 are in good agreement with those of recent slip line studies performed on CP Ti specimens with slightly different compositions (Li et al., 2013; Barkia et al., 2015). Only a lower frequency of grains with primary basal slip may be noticed for the present data ( 0 to 1\% in Tables 4 and 5 to be compared with 5 to $10 \%$ found by Li et al. (2013); Barkia et al. (2015)). It is also important to note that, while these results provide a qualitative basis for proposing a relevant model of plasticity of Ti in tensile condition, one should not seek to quantitatively reproduce in the bulk the data of surface observations in Tables 4 and 5 . Indeed, because of boundary conditions and possible gradients in material composition, the distribution of slip system activities at the sample surface may not be perfectly representative of the distribution in the bulk. Furthermore, slip systems with Burgers vectors nearly parallel to the surface may be underestimated compared to those with Burgers vectors nearly normal to the surface. Some slip systems may also be missed because of diffuse slip leading to not well-defined slip bands (Li et al., 2013). Uncertainty in slip families identification may especially arise because several slip planes may be associated to very close slip traces. In particular, the estimated proportion of $\Pi_{1}$ planes compared to $\Pi_{2}$ planes may not be significant since these two planes often had very close slip traces in the investigated specimens.

\section{Micromechanical modeling}

\subsection{Single crystal constitutive laws}

The behavior of $\mathrm{CP}$ Ti is supposed to be elastic-viscoplastic. Following our conjecture that twinning plays only a secondary role in the deformation behavior of our samples, twinning is not considered in the model. Only glide on the 5 slip families listed in Table 2 is taken into account to achieve viscoplastic strain. At the grain level, the viscoplastic strain rate tensor $\dot{\varepsilon}_{i j}^{p}$ and the viscoplastic spin tensor $\dot{\omega}_{i j}^{p}$ are thus provided by

$$
\dot{\varepsilon}_{i j}^{p}=\sum_{s} R_{i j}^{(s)} \dot{\gamma}^{(s)}, \quad \dot{\omega}_{i j}^{p}=\sum_{s} S_{i j}^{(s)} \dot{\gamma}^{(s)}
$$

where $\dot{\gamma}^{(s)}, R_{i j}^{(s)}$ and $S_{i j}^{(s)}$ are, respectively, the slip rate, the symmetric and the skew-symmetric Schmid orientation tensors associated to system $(s) \cdot \dot{\gamma}^{(s)}$ is given through the Orowan relation

$$
\dot{\gamma}^{(s)}=\rho_{m}^{(s)} b^{(s)} v^{(s)}
$$

where $\rho_{m}^{(s)}, b^{(s)}$ and $v^{(s)}$ are, respectively, the mobile dislocation density, the Burgers vector magnitude and the average dislocation velocity associated to system $(s)$. Hence, the present model deals separately with mobile dislocation density and dislocation velocity which, in contrast, are usually merged in standard polycrystal plasticity treatment (e.g., Salem et al. (2006); Knezevic et al. (2013)). This feature allows us to consider the possibility of mobile dislocation multiplication, as suggested by Conrad (1981) and Naka (1983). The conjecture is that the rapid multiplication of mobile dislocations will induce a drop in their average glide velocity, which will considerably reduce the increase of the flow stress and will end up to a low work hardening stage A. The average dislocation velocity on system $(s)$ is assumed to follow a classic power law relationship

$$
v^{(s)}=v_{0}^{(s)}\left|\frac{\tau^{(s)}}{\tau_{c}^{(s)}}\right|^{n^{(s)}} \operatorname{sgn}\left(\tau^{(s)}\right)
$$

where $v_{0}^{(s)}$ is a reference velocity, $n^{(s)}$ is the inverse of the SRSS, $\tau^{(s)}$ is the resolved shear stress $\left(\tau^{(s)}=R_{i j}^{(s)} \sigma_{i j}\right)$ and $\operatorname{sgn}\left(\tau^{(s)}\right)$ is the sign of $\tau^{(s)} \cdot \tau_{c}^{(s)}$ stands for the CRSS. It writes as the sum of the lattice friction stress $\tau_{0}^{(s)}$ and an expanded Taylor relation that accounts for dislocation interactions between systems (Franciosi et al., 1980)

$$
\tau_{c}^{(s)}=\tau_{0}^{(s)}+\mu^{(s)} b^{(s)} \sqrt{\sum_{l} a^{(s l)} \rho_{f}^{(l)}}
$$

$a^{(s l)}$ denotes the interaction coefficient which is related to the strength of the interaction between system $(s)$ and $(l) . \mu^{(s)}$ is the directional shear modulus of system $(s) . \rho_{f}^{(l)}$ depicts forest (or sessile) dislocation density on system $(l)$. The evolution equations for the two dislocation density species are adopted following indirectly the original work of Estrin and Kubin (1986).

$$
\dot{\rho}_{m}^{(s)}=\frac{1}{b^{(s)}}\left[\frac{C_{1}^{(s)}}{b^{(s)}}-\frac{1}{L^{(s)}}\right]\left|\dot{\gamma}^{(s)}\right|
$$




$$
\dot{\rho}_{f}^{(s)}=\frac{1}{b^{(s)}}\left[\frac{1}{L^{(s)}}-2 k_{c}^{(s)} b^{(s)} \rho_{f}^{(s)}\right]\left|\dot{\gamma}^{(s)}\right|
$$

It is seen that the negative terms in Eq. (6), which correspond to mobile dislocation immobilization, appear as positive terms in Eq. (7) and stand for storage of forest dislocations. The mean free path of mobile dislocations $L^{(s)}$ is assumed to depend on reactions with other mobile dislocations, which are considered through the parameter $C_{2}^{(s)}$, and interactions with forest dislocations, which are taken into account through the interaction coefficients $a^{(s l)}$ and the constant $K^{(s)}$

$$
\frac{1}{L^{(s)}}=b^{(s)} C_{2}^{(s)} \rho_{m}^{(s)}+\frac{\sqrt{\sum_{l} a^{(s l)} \rho_{f}^{(l)}}}{K^{(s)}}
$$

$C_{1}^{(s)}$ is related with mobile dislocations production. $k_{c}^{(s)} b^{(s)}$ represents the annihilation distance between dislocations and the term associated to it thus accounts for dynamic recovery.

\subsection{Micro-macro scale transition}

The scale transition scheme used in the present study relies on the very recent work of Mareau and Berbenni (2015) but, here, thermal strains are disregarded. These authors proposed a new homogenization model based on an affine linearization of the viscoplastic flow rule and the translated fields technique to formulate the 1-site self-consistent approximation. The initial translated field technique was actually developed assuming a secant linearization for viscoplatic strain rates (Paquin et al., 1999) and was first applied to polycrystalline steels by Paquin et al. (2001). It was shown by Mareau and Berbenni (2015) that a better accuracy was obtained with an affine linearization instead of a secant one regarding macroscopic and local (grain level) responses compared to full field FFT simulations. The model of Mareau and Berbenni (2015) provides same results as the hereditary approach of Masson and Zaoui (1999) but its numerical implementation is much easier since, as an internal variable approach, no use of Laplace-Carson transform is needed. Here, the main steps are recalled. They include the field equations of the problem, its integral equation, a qualitative description of the translated fields technique and use of selfconsistent approximations. At the end of the subsection, the interaction law for stresses is given.

\subsubsection{Field equations and integral equation}

In homogenization theory, the macroscopic stress rate and strain rate tensors ( $\dot{\boldsymbol{\Sigma}}$ and $\dot{\mathbf{E}})$ of a representative volume element $V$ are obtained by volume averaging the local stress rate and strain rate tensors ( $\dot{\boldsymbol{\sigma}}$ and $\dot{\boldsymbol{\varepsilon}}$ ) as follows

$$
\dot{\boldsymbol{\Sigma}}=\frac{1}{V} \int_{V} \dot{\boldsymbol{\sigma}}(\boldsymbol{x}) \boldsymbol{d} \boldsymbol{x}=\left\langle\dot{\boldsymbol{\sigma}}>, \dot{\boldsymbol{E}}=\frac{1}{V} \int_{V} \dot{\boldsymbol{\varepsilon}}(\boldsymbol{x}) \boldsymbol{d} \boldsymbol{x}=\langle\dot{\boldsymbol{\varepsilon}}\rangle\right.
$$

Within the small strain framework, the local total strain rate relative to an elastic-viscoplastic behavior splits into an elastic part and a viscoplastic one, $\dot{\boldsymbol{\varepsilon}}=\dot{\boldsymbol{\varepsilon}}^{\boldsymbol{e}}+\dot{\boldsymbol{\varepsilon}}^{\boldsymbol{p}}$. The linear Hooke's law relates $\dot{\boldsymbol{\varepsilon}}^{\mathbf{e}}$ to the local Cauchy stress rate $\dot{\boldsymbol{\sigma}}, \dot{\boldsymbol{\varepsilon}}^{\boldsymbol{e}}=\boldsymbol{s}: \dot{\boldsymbol{\sigma}}$, where the colon : denotes the contracted product of two tensors, and $\boldsymbol{s}$ (with $\boldsymbol{c}=\boldsymbol{s}^{-1}$ ) is the local elastic compliance tensor. Using a first order affine linearization, the viscoplastic strain rate is written $\dot{\boldsymbol{\varepsilon}}^{\boldsymbol{p}}=\boldsymbol{m}: \boldsymbol{\sigma}+\dot{\boldsymbol{\eta}}$ where $\boldsymbol{m}$ (with $\boldsymbol{b}=\boldsymbol{m}^{-1}$ ) is the tangent viscoplastic compliance tensor defined by $\boldsymbol{m}=\frac{\partial \dot{\varepsilon}^{p}}{\partial \boldsymbol{\sigma}}$, and $\dot{\boldsymbol{\eta}}$ corresponds to a back-extrapolated strain rate. According to the constitutive power law used in the present study (Eqs. (2)-(4)), the components of the tangent viscoplastic compliance tensor and of the back-extrapolated strain rate tensor write, respectively, as

$$
\begin{aligned}
& m_{i j k l}=\sum_{s} n^{(s)} \rho_{m}^{(s)} b^{(s)} v_{0}^{(s)} \frac{\left|\tau^{(s)}\right|^{n^{(s)}-1}}{\left|\tau_{c}^{(s)}\right|^{n^{(s)}}} R_{i j}^{(s)} R_{k l}^{(s)} \\
& \dot{\eta}_{i j}=\sum_{s}\left(1-n^{(s)}\right) \rho_{m}^{(s)} b^{(s)} v_{0}^{(s)}\left|\frac{\tau^{(s)}}{\tau_{c}^{(s)}}\right|^{n^{(s)}} \operatorname{sgn}\left(\tau^{(s)}\right) R_{i j}^{(s)}
\end{aligned}
$$

In the absence of volume forces, static equilibrium conditions impose $\operatorname{div} \boldsymbol{\sigma}=0$ and $\operatorname{div} \boldsymbol{\sigma}=0$, whereas kinematic compatibility gives $\dot{\boldsymbol{\varepsilon}}=\frac{1}{2}\left(\nabla \dot{\boldsymbol{u}}+{ }^{t} \nabla \dot{\boldsymbol{u}}\right)$, with $\dot{\boldsymbol{u}}$ is the velocity field. On the boundary $\partial V$ of $V$, a prescribed displacement is considered, $\dot{\boldsymbol{u}}(\boldsymbol{x})=\dot{\boldsymbol{E}} \cdot \boldsymbol{x}$ on $\partial V$.

In order to recast the heterogeneous problem given by the preceding field equations in the form of a single integral equation, a homogeneous reference medium with uniform elastic moduli $\boldsymbol{C}$ (respectively, uniform elastic compliances $\boldsymbol{S}=\boldsymbol{C}^{-1}$ ), uniform tangent viscoplastic moduli $\boldsymbol{B}$ (respectively, uniform viscoplastic compliances $\boldsymbol{M}=\boldsymbol{B}^{-1}$ ) and uniform backextrapolated strain rates $\dot{\boldsymbol{N}}$, is introduced. The local tensors $\boldsymbol{c}, \boldsymbol{s}, \boldsymbol{b}, \boldsymbol{m}$ and $\dot{\boldsymbol{\eta}}$ can then be expressed as the sum of a uniform reference tensor (respectively, $\boldsymbol{C}, \boldsymbol{S}, \boldsymbol{B}, \boldsymbol{M}$ and $\dot{\boldsymbol{N}}$ ) and a fluctuating part (respectively, $\boldsymbol{\delta} \boldsymbol{c}, \boldsymbol{\delta} \boldsymbol{s}, \boldsymbol{\delta b}, \boldsymbol{\delta} \boldsymbol{m}$ and $\boldsymbol{\delta} \dot{\boldsymbol{\eta}}$ ) 


$$
\begin{aligned}
\boldsymbol{c}(\boldsymbol{x}) & =\boldsymbol{C}+\boldsymbol{\delta} \boldsymbol{c}(\boldsymbol{x}), \quad \boldsymbol{s}(\boldsymbol{x})=\boldsymbol{S}+\boldsymbol{\delta} \boldsymbol{s}(\boldsymbol{x}) \\
\boldsymbol{b}(\boldsymbol{x}) & =\boldsymbol{B}+\boldsymbol{\delta} \boldsymbol{b}(\boldsymbol{x}), \quad \boldsymbol{m}(\boldsymbol{x})=\boldsymbol{M}+\boldsymbol{\delta} \boldsymbol{m}(\boldsymbol{x}) \\
\dot{\boldsymbol{\eta}}(\boldsymbol{x}) & =\dot{\boldsymbol{N}}+\boldsymbol{\delta} \dot{\boldsymbol{\eta}}(\boldsymbol{x})
\end{aligned}
$$

In order to obtain the integral equation, the symmetric modified Green operators $\boldsymbol{\Gamma}^{\boldsymbol{C}}$ and $\boldsymbol{\Gamma}^{\boldsymbol{B}}$ are introduced as defined by Kröner (1989). Here, they are associated to the elastic and viscoplastic reference media, respectively (see their expressions in the paper of Mareau and Berbenni (2015)). Then, the expression of the integral equation is deduced by considering the specific properties of the modified Green operators for any kinematic compatible fields and statically balanced fields (Kröner, 1989) in combination with the set of field equations

$$
\dot{\boldsymbol{\varepsilon}}=\dot{\boldsymbol{E}}-\Gamma^{\boldsymbol{C}} * \delta \boldsymbol{c}: \dot{\boldsymbol{\varepsilon}}^{\boldsymbol{e}}-\Gamma^{B}\left(\delta \boldsymbol{b}:\left(\dot{\boldsymbol{\varepsilon}}^{p}-\dot{\boldsymbol{\eta}}\right)-\boldsymbol{B}: \delta \dot{\eta}\right)+\left(\Gamma^{\boldsymbol{C}}: \boldsymbol{C}-\Gamma^{\boldsymbol{B}}: \boldsymbol{B}\right){ }^{*} \dot{\boldsymbol{\varepsilon}}^{p}
$$

where the symbol * denotes the spatial convolution product.

\subsubsection{Translated fields technique and self-consistent approximation}

In the resolution scheme based on the translated fields technique (Paquin et al., 1999), the purely elastic and purely viscoplastic problems are first resolved independently using self-consistent approximations. The main idea of self-consistent approximation is to weaken the non-local part of the modified Green operators, that decreases as $\frac{1}{\text { I }^{\prime}}$ (Kröner, 1989), by imposing averaging conditions such that the non-local interactions can finally be neglected. Given the $\boldsymbol{x}^{\boldsymbol{x}} \boldsymbol{x}^{\prime} \mathbf{v}^{3}$ (Kaging conditions on $\dot{\boldsymbol{\Sigma}}$ and $\dot{\boldsymbol{E}}$ (Eq. (9)), the self-consistent approximations actually impose that the effective medium is considered as the homogeneous reference medium. The self-consistent solutions of the purely elastic and purely viscoplastic problems are then, respectively, given by Mareau and Berbenni (2015)

$$
\dot{e}^{e}=A^{C^{e}}: \dot{E}, \dot{e}^{p}=A^{B^{e}}: \dot{E}+A^{B^{e}}: \Gamma_{l}^{B^{e}}:\left(b: \dot{\eta}-B^{e}: \dot{N}^{e}\right)
$$

where $\boldsymbol{A}^{\boldsymbol{C}^{\boldsymbol{e}}}=\left(\boldsymbol{I}+\boldsymbol{\Gamma}_{\boldsymbol{l}}^{\boldsymbol{C}^{\boldsymbol{e}}}: \delta \boldsymbol{c}\right)^{-1}$ and $\boldsymbol{A}^{\boldsymbol{B}^{\boldsymbol{e}}}=\left(\boldsymbol{I}+\boldsymbol{\Gamma}_{\boldsymbol{l}}^{\boldsymbol{B}^{\boldsymbol{e}}}: \boldsymbol{\delta} \boldsymbol{b}\right)^{-1}$ (with $\boldsymbol{I}$ the fourth order unit tensor) are the strain rate concentration tensors associated, respectively, to the effective elastic and viscoplastic media. $\boldsymbol{\Gamma}_{\boldsymbol{l}}^{\boldsymbol{C}^{\boldsymbol{e}}}$ and $\boldsymbol{\Gamma}_{\boldsymbol{l}}^{\boldsymbol{B}^{\boldsymbol{e}}}$ are the local parts of $\boldsymbol{\Gamma}^{\boldsymbol{C}}$ and $\boldsymbol{\Gamma}^{\boldsymbol{B}^{\boldsymbol{e}}}$. Besides, the resolution of the purely viscoplastic problem allows to deduce the effective back-extrapolated strain rate tensor $\dot{\boldsymbol{N}}^{\boldsymbol{e}}$ as

$$
\dot{\boldsymbol{N}}^{\boldsymbol{e}}=\boldsymbol{M}^{\boldsymbol{e}}:\left(<\boldsymbol{b}: \boldsymbol{A}^{\boldsymbol{B}^{\boldsymbol{e}}}: \boldsymbol{\Gamma}_{\boldsymbol{l}}^{\boldsymbol{B}^{\boldsymbol{e}}}>-\boldsymbol{I}\right)^{-1}:<\boldsymbol{b}: \boldsymbol{A}^{\boldsymbol{B}^{\boldsymbol{e}}}: \boldsymbol{\Gamma}_{\boldsymbol{l}}^{\boldsymbol{B}^{\boldsymbol{e}}}: \boldsymbol{b}: \dot{\boldsymbol{\eta}}-\boldsymbol{b}: \dot{\boldsymbol{\eta}}>
$$

The resolution of the heterogeneous problem is then carried out by introducing translated fields (Paquin et al., 1999; Mareau and Berbenni, 2015). In this approach, $\dot{\boldsymbol{\varepsilon}}^{\boldsymbol{e}}$ and $\dot{\boldsymbol{\varepsilon}}^{\boldsymbol{p}}$ are decomposed into the kinematically admissible fields, $\dot{\boldsymbol{e}}^{\boldsymbol{e}}$ and $\dot{\mathbf{e}}^{\mathbf{p}}$, and residual fields which are associated to fictitious translated balanced stress rate fields, $\dot{\boldsymbol{\sigma}}^{\prime}$ and $\dot{\boldsymbol{\sigma}}^{\prime \prime}$, as follows $\dot{\boldsymbol{\varepsilon}}^{\boldsymbol{e}}=\dot{\boldsymbol{e}}^{\boldsymbol{e}}+\boldsymbol{s}$ : $\dot{\boldsymbol{\sigma}}^{\prime}$ and $\dot{\boldsymbol{\varepsilon}}^{\boldsymbol{p}}=\dot{\boldsymbol{e}}^{\boldsymbol{p}}+\boldsymbol{s}: \dot{\boldsymbol{\sigma}}^{\prime \prime}$. The translated strain rate field $\dot{\boldsymbol{\varepsilon}}^{\star}$ is then defined as $\dot{\boldsymbol{\varepsilon}}^{\star}=\dot{\boldsymbol{\varepsilon}}-\dot{\boldsymbol{e}}^{\boldsymbol{e}}-\dot{\boldsymbol{e}}^{\boldsymbol{p}}=\boldsymbol{s}: \dot{\boldsymbol{\sigma}}^{\prime}+\boldsymbol{s}: \dot{\boldsymbol{\sigma}}^{\prime \prime} . \dot{\boldsymbol{\varepsilon}}^{\star}$ is a kinematic compatible field, which is of great interest for taking advantage of specific properties of modified Green operators. It can be shown that $\dot{\boldsymbol{\varepsilon}}^{\star}$ is the solution of a translated problem described by the following integral equation (Mareau and Berbenni, 2015)

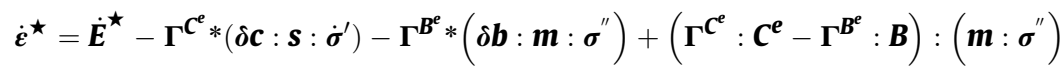

where $\dot{\boldsymbol{E}}^{\star}=\left\langle\dot{\boldsymbol{\varepsilon}}^{\star}\right\rangle$. Then, similar procedures as before are applied in order to get a self-consistent approximation of $\dot{\boldsymbol{\varepsilon}}^{\star}$. The local strain rate $\dot{\boldsymbol{\varepsilon}}$ is accordingly given by the relation $\dot{\boldsymbol{\varepsilon}}=\dot{\boldsymbol{\varepsilon}}^{\star}+\dot{\boldsymbol{e}}^{\boldsymbol{e}}+\dot{\boldsymbol{e}}^{\boldsymbol{p}}$ and, finally, the expression of the interaction law for stresses is obtained (Mareau and Berbenni, 2015)

$$
\begin{aligned}
\dot{\sigma} & =\boldsymbol{c}: A^{C^{e}}:\left(S^{e}: \dot{\Sigma}+\dot{E}_{p}^{e}\right)-c: \dot{\varepsilon}^{p}+c: A^{C^{e}}: \Gamma_{l}^{B^{e}}:\left(b: \dot{\eta}-B^{e}: \dot{N}^{e}\right)+c: A^{C^{e}}: \Gamma_{l}^{C^{e}}:\left(\delta c: \dot{\varepsilon}^{p}+c: A^{C^{e}}\right. \\
& \left.:\left(<\dot{\varepsilon}^{p}>-\dot{E}_{p}^{e}\right)\right)-c: A^{C^{e}}: \Gamma_{l}^{B^{e}}:\left(\delta b: \dot{\varepsilon}^{p}-b: A^{B^{e}}:\left(M^{e}: \Sigma+\dot{N}^{e}-<\dot{\varepsilon}^{p}\right)\right)+c: A^{C^{e}}:\left(\Gamma_{l}^{C^{e}}: C^{e}-\Gamma_{l}^{B^{e}}: B^{e}\right) \\
& :\left(\dot{\varepsilon}^{p}-A^{B^{e}}:<\dot{\varepsilon}^{p}>\right)-c: A^{C^{e}}:\left(\Gamma_{l}^{C^{e}}: C^{e}-\Gamma_{l}^{B^{e}}: B^{e}\right):\left(A^{B^{e}}: \Gamma_{l}^{B^{e}}:\left(b: \dot{\eta}-B^{e}: \dot{N}^{e}\right)\right)
\end{aligned}
$$

where $\dot{\boldsymbol{E}}_{\boldsymbol{p}}^{\boldsymbol{e}}$ is the effective viscoplastic strain rate computed to satisfy the averaging condition on $\dot{\boldsymbol{\Sigma}}$ (Eq. (9)) (see the paper of Mareau and Berbenni (2015) for the explicit expression). 

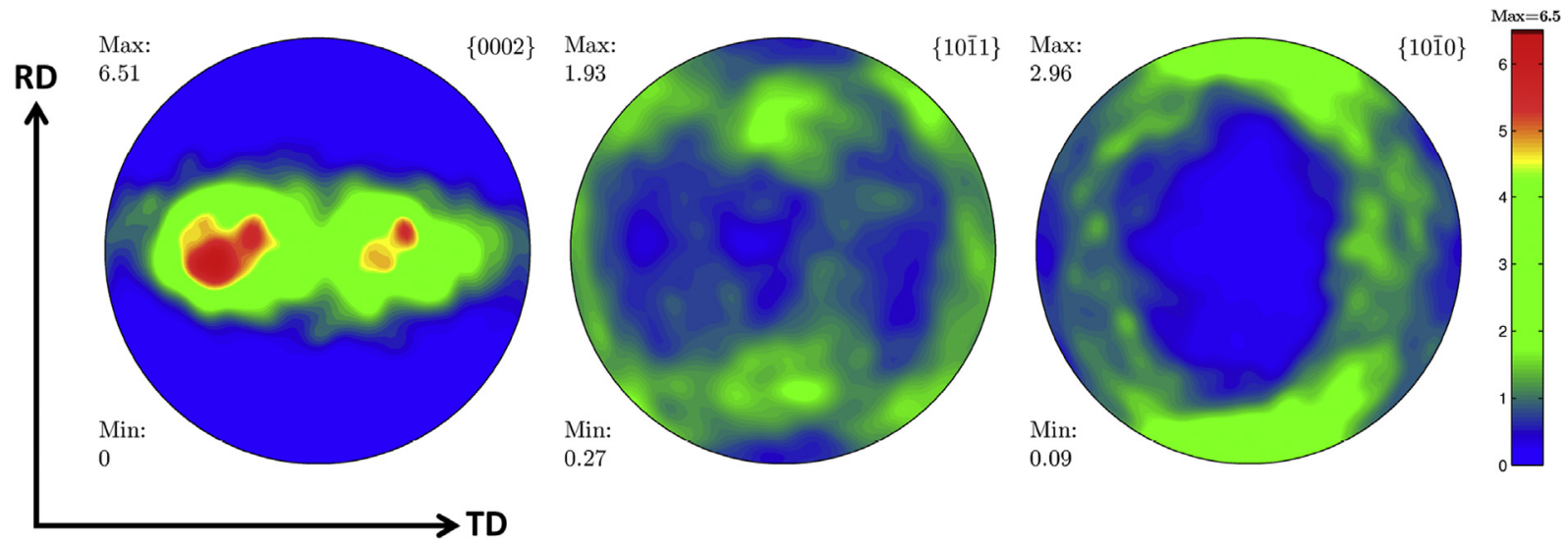

Fig. 2. Pole figures obtained from the initial 5547 grain orientations weighted by their volume fraction that are considered in the model. The plots were performed with the MTEX toolbox (Bachmann et al., 2010).

\subsection{Description and evolution of the microstructure}

In the model, each point $\boldsymbol{x}$ represents a grain to which is associated a mean crystallographic orientation, a morphology, and a volume fraction. The modified Green operators are hence integrated over each grain volume. In this study, all grains are assumed of spherical shape. Initial EBSD measurements have allowed to fix a list of 5547 grains with equivalent diameter greater than $2 \mu \mathrm{m}$ that constitutes the representative volume element $V$. The pole figures obtained from these 5547 orientations (see Fig. 2) are indeed pretty similar as the ones measured from 708,771 orientations in Fig. 1.

Within the present small strain setting, the updating of slip system directions $\boldsymbol{u}^{(\boldsymbol{s})}$ and slip plane normals $\boldsymbol{v}^{(\boldsymbol{s})}$ result only from the elastic rotation tensor $\omega^{\boldsymbol{e}}$

$$
\begin{aligned}
& \boldsymbol{u}^{(\boldsymbol{s})}=\left(\boldsymbol{I}+\omega^{\boldsymbol{e}}\right) \cdot \boldsymbol{u}^{0(\boldsymbol{s})} \\
& \boldsymbol{v}^{(\boldsymbol{s})}=\boldsymbol{v}^{0(\boldsymbol{s})} \cdot\left(\boldsymbol{I}+\omega^{\boldsymbol{e}}\right)^{-1}
\end{aligned}
$$

where $\boldsymbol{u}^{0(\boldsymbol{s})}$ and $\boldsymbol{v}^{0(\boldsymbol{s})}$ are the slip directions and slip plane normals in the undeformed state. Disregarding the effect of heterogeneous elasticity on rotations, $\omega^{\boldsymbol{e}}=-\omega^{\boldsymbol{p}}$ for grains of spherical shape, $\omega^{\boldsymbol{p}}$ being the viscoplastic rotation tensor (see the expression of its time derivative in Eq. (2)).

\subsection{Identification of model parameters}

Table 6 provides the model parameters that are related to the Ti crystal lattice at room temperature. For each slip family, the directional shear modulus $\mu^{(s)}$ is computed from the elastic compliances matrix rotated in a frame attached to one slip system of the family. The choice to consider directional shear moduli in the Taylor relation (Eq. (5)) is motivated by the fact that it allows accounting partially of the strong anisotropy of the CRSSs thanks to physically-based and non-fitted parameters. Indeed, shear moduli are the lowest on $P$ and $\Pi_{1}^{<a>}$ systems and about 1.4 higher on $B, \Pi_{1}^{<c+a>}$ and $\Pi_{2}^{<c+a>}$ systems (Table 6). This tendency is qualitatively similar to the estimates of CRSSs that can be found in the literature, as can be seen in Table 7. In particular, the consideration of elastic anisotropy in the Taylor relation may have some importance concerning $B$ slip since $a b$ initio and Molecular Dynamics approaches on pure Ti do not report very strong differences between $P$ and $B$ lattice friction stresses (Poty et al., 2011). However, most homogenization models have to consider strong differences between CRSSs in order to fit experimental observations (Philippe et al., 1995; Salem et al., 2005; Wu et al., 2007; Gurao et al., 2011; Warwick et al., 2012; Benmhenni et al., 2013; Gloagen et al., 2013; Knezevic et al., 2013) (Table 7). From Table 7, it can be noted that

Table 6

Model parameters related to the Ti crystal lattice at room temperature. The values of $c / a$ and $b^{<a>}$ are taken from Nemat-Nasser et al. (1999) and the values of

\begin{tabular}{|c|c|c|c|c|}
\hline$c / a$ & $b^{<a>}$ & & $b^{<c+a>}$ & \\
\hline 1.587 & $2.95 \AA$ & & $5.53 \AA$ & \\
\hline $\begin{array}{l}C_{11} \\
160 \mathrm{GPa}\end{array}$ & $\begin{array}{l}C_{33} \\
181 \mathrm{GPa}\end{array}$ & $\begin{array}{l}C_{44} \\
46.5 \mathrm{GPa}\end{array}$ & $\begin{array}{l}C_{12} \\
90 \mathrm{GPa}\end{array}$ & $\begin{array}{l}C_{13} \\
66 \mathrm{GPa}\end{array}$ \\
\hline$\mu^{P}$ & $\mu^{B}$ & $\mu^{\Pi_{1}^{(a)}}$ & $\mu^{\Pi_{1}^{(c+a)}}$ & $\mu^{\Pi_{2}^{\langle c+a\rangle}}$ \\
\hline $35.0 \mathrm{GPa}$ & $46.5 \mathrm{GPa}$ & $37.1 \mathrm{GPa}$ & $47.7 \mathrm{GPa}$ & $49.2 \mathrm{GPa}$ \\
\hline
\end{tabular}
stiffness constants from Hearmon (1979). 
Non-exhaustive literature data for CRSS ratios relative to $P$ slip in CP or highly pure $\alpha$-Ti at room temperature. For $P$ systems, the absolute values of CRSS are given. The CRSSs stand for to the values of resolved shear stress when slip initiates on a system. For the present study and for the model of Benmhenni et al. (2013), they thus correspond to $\tau_{\mathbf{c}}^{(\mathbf{s})}$ computed from initial dislocation densities (Eq. (5)). Values used in the present study are written in bold.

\begin{tabular}{|c|c|c|c|c|c|c|c|}
\hline Method & $P$ & $B$ & $\Pi_{1}^{\langle a\rangle}$ & $\Pi_{1}^{\langle c+a\rangle}$ & $\Pi_{2}^{\langle c+a\rangle}$ & $\mathrm{O}(\mathrm{wt} . \%)$ & Ref. \\
\hline \multirow[t]{3}{*}{ Slip line analyses } & $90 \mathrm{MPa}$ & 1.2 & 1.1 & - & - & 0.10 & Churchman (1954) \\
\hline & $14 \mathrm{MPa}$ & 3.0 & - & - & - & 0.01 & \\
\hline & - & 3.6 & 25.4 & 22.5 & - & 0.25 & Li et al. (2013) \\
\hline TEM observations & - & - & $<13$ & - & - & 0.10 & Zaefferer (2003) \\
\hline \multirow[t]{2}{*}{ Slip line analyses + TEM observations } & $120 \mathrm{MPa}$ & 1.5 & 1.2 & 2 & - & 0.16 & Barkia et al. (2015) \\
\hline & $192 \mathrm{MPa}$ & 1.5 & 1.2 & 1.8 & - & 0.32 & \\
\hline \multirow[t]{2}{*}{ FEM modeling of single crystals } & $181 \mathrm{MPa}$ & 1.2 & - & 2.6 & - & 0.07 & Gong and Wilkinson (2009) \\
\hline & $150 \mathrm{MPa}$ & 2.3 & - & 7.4 & - & & Zambaldi et al. (2012) \\
\hline \multirow[t]{3}{*}{ Taylor-type modeling } & & 6.0 & - & 9.0 & - & 0.11 & Philippe et al. (1995) \\
\hline & $37 \mathrm{MPa}$ & 1.3 & - & 5.3 & - & $<0.0002$ & Salem et al. (2005) \\
\hline & $30 \mathrm{MPa}$ & 5.0 & - & 4.0 & - & $<0.0002$ & Wu et al. (2007) \\
\hline \multirow[t]{6}{*}{ Self-consistent modeling } & $90 \mathrm{MPa}$ & 2.5 & - & 9.4 & - & & Gurao et al. (2011) \\
\hline & $80 \mathrm{MPa}$ & 1.1 & 1.4 & 3.3 & - & 0.06 & Warwick et al. (2012) \\
\hline & $57 \mathrm{MPa}$ & 4.8 & 2.9 & 5.4 & 4.1 & 0.13 & Benmhenni et al. (2013) \\
\hline & $68 \mathrm{MPa}$ & 2.6 & 1.8 & 3.7 & - & 0.12 & Gloagen et al. (2013) \\
\hline & $98 \mathrm{MPa}$ & - & - & 2.3 & - & $<0.001$ & Knezevic et al. (2013) \\
\hline & $141 \mathrm{MPa}$ & 1.8 & 1.4 & 2.4 & 2.5 & 0.11 & Present study \\
\hline
\end{tabular}

$\Pi_{2}^{\langle c+a\rangle}$ slip family is seldom taken into consideration in the models. $\Pi_{2}^{\langle c+a\rangle}$ slip has nevertheless been unambiguously observed in Ti, in particular, in single crystals loaded parallel to their $\langle c>$ axis (Minonishi et al., 1982; Xiaoli et al., 1994). It is also reported that $\Pi_{2}^{<c+a>}$ glide should be the most difficult one (Xiaoli et al., 1994).

According to the single crystal constitutive laws, the model would require to fit 9 parameters per slip family in addition to the interaction coefficients $a_{i j}$. In order to handle a limited total number of parameters, only $\tau_{0}, C_{1}$ and $n$ are supposed to depend on slip family. First, specific values for the 5 slip families are considered for the lattice friction stress since, as explained above, the anisotropy of dislocations glide is well-documented for Ti (Table 7). Furthermore, the results of crystal plasticity models are known to be very sensitive to the different CRSS values (Philippe et al., 1995; Salem et al., 2005; Wu et al., 2007; Gong and Wilkinson, 2009; Gurao et al., 2011; Warwick et al., 2012; Benmhenni et al., 2013; Gloagen et al., 2013; Knezevic et al., 2013). Within our constitutive setting, the anisotropy of slip can also result from dislocation densities evolution. Therefore, it is supposed that the constant $C_{1}$, which is linked to mobile dislocations multiplication, has specific values for the $P$ family. Assuming a higher $C_{1}$ value for $P$ systems allows having a higher total dislocation density in RD-samples since the slip lines analysis has revealed that they deform more frequently on $P$ systems than TD-samples. Emphasizing the differences of dislocation density between orientations helps reproducing the higher work hardening rate observed along RD during stage $C$, which is difficult to retrieve otherwise. Finally, following our initial hypothesis explaining the strain rate effect on the three-stage work hardening behavior, a specific SRSS for the $P$ family is also assumed (see Table 8 ). All the other parameters are taken equal for all slip systems (see Table 9).

It is a hard task to evaluate quantitatively the interaction coefficients $a_{i j}$. Discrete dislocation dynamics (DDD) simulations have allowed progress in this domain by computing quantitative values of the hardening coefficients, initially for facecentered cubic metals by Madec et al. (2003); Hoc et al. (2004); Devincre et al. (2005, 2006); Kubin et al. (2008), then for body-centered cubic metals by Queyreau et al. (2009) and more recently for some hexagonal materials (ice by Devincre (2013) and magnesium by Bertin et al. (2014)). All these studies underline the very strong value of the coefficient pertaining to collinear interactions, i.e. reactions between dislocations with parallel Burgers vectors gliding in different planes. Other interactions are found to be close, except for the self-hardening coefficients which might be one order of magnitude smaller (Hoc et al., 2004). However, if the probability of double cross-slip of screw dislocations is taken into account, it is shown that an effective self-interaction coefficient must be considered instead, because of the strong collinear interactions between primary screw dislocations and edge jogs left on cross-slip systems (Hoc et al., 2004; Devincre, 2013). The effective selfinteraction coefficient is thus typically of the same order as the latent coefficients related to non-collinear interactions (Hoc et al., 2004; Devincre, 2013). No similar DDD studies were realized for Ti but cross-slip mechanisms were experimentally observed (Naka, 1983; Naka and Lasalmonie, 1983; Chichili et al., 1998). As a consequence, two different values of interaction coefficients are considered in the present model, one for the collinear interactions $a_{\text {coli }}=0.7$ and another for the other kinds of interactions $a_{\neq \text {coli }}=0.1$. These are typical values obtained by DDD simulations (Madec et al., 2003; Hoc et al., 2004; Devincre et al., 2005, 2006; Kubin et al., 2008; Queyreau et al., 2009; Devincre, 2013; Bertin et al., 2014). In addition to being physically justified by DDD simulations, the choice of strong collinear interaction coefficients enables reinforcing the anisotropy between $P, B$ and $\Pi_{1}^{\langle a\rangle}$ families. Indeed, according to Eq. (5), the growth of dislocation density on the first activated system (typically a $P$ one) will thus stronger harden its collinear systems (belonging to $B$ or $\Pi_{1}^{<a>}$ families) than itself.

The model comprises 17 parameters to fit. The fitting procedure was performed such that the parameters remain within realistic bounds since they all have clear physical meaning. In particular, it was checked that the obtained dislocation densities and mean free paths are credible. The genetic algorithm provided in Matlab was used to perform a first identification of the parameters, based on the best possible fit of 6 experimental stress-strain curves (RD and TD directions both at initial strain 
Model parameters that are specific to slip families.

\begin{tabular}{lllll}
\hline & $P$ & $\Pi_{1}^{<a>}$ & $\boldsymbol{B}$ & $\Pi_{1}^{\langle c+a\rangle}$ \\
\hline$\tau_{0}$ & $90 \mathrm{MPa}$ & $140 \mathrm{MPa}$ & $180 \mathrm{MPa}$ & $210 \mathrm{MPa}$ \\
$C_{1}$ & $8 \cdot 10^{-4}$ & & $1.5 \cdot 10^{-4}$ \\
$n$ & 65 & & 32 & $225 \mathrm{MPa}$ \\
\hline
\end{tabular}

rates of $8.10^{-3} \mathrm{~s}^{-1}, 5.10^{-4} \mathrm{~s}^{-1}$ and $5.10^{-5} \mathrm{~s}^{-1}$ taken from Roth et al. (2014)). Then, the definitive set of parameters was adjusted searching to reproduce qualitatively the characteristic features found experimentally:

- a three-stage work hardening and, importantly, the inversion of the sign of the strain rate effect with regard to the orientation of the tensile axis, as observed by Roth et al. (2014),

- a realistic activity of $P$ systems,

- correct values of Lankford coefficients,

- qualitatively consistent textures evolution.

\section{Results}

\subsection{Work hardening behavior and stress-strain curves}

Fig. 3 compares model predictions with the experimental measurements of the work hardening rate evolution (Roth et al., 2014). It is seen that the three-stage character of the work hardening behavior is indeed qualitatively retrieved. The striking confirmation that the model correctly seizes the physical mechanisms stems from the observation of an opposite strain rate effect on the well depth for RD and TD orientations. The quantitative agreement is much better for TD specimens, where the levels of strain hardening and plastic strain corresponding to the local $\Theta$ minimum are close to the experimental values. The simulated $\Theta$ increments during stages B are however less pronounced than the measured ones. For RD specimens, the work hardening rate during stage $B$ is much higher than that observed experimentally. Nevertheless, $\Theta$ increments remain quantitatively consistent with the measurements. In particular, concerning the sample deformed at the highest strain rate, the work hardening rate evolution does not display a well but an inflexion point, similarly to experiment.

The variation of the model parameters allowed for picking out those controlling the seeked qualitative features. These checks showed that indeed, the suggestion of different values for the SRSS and the dislocation multiplication rate in the prismatic and pyramidal glide systems plays a crucial role for reproducing the experimentally observed behavior. To illustrate this statement, insets in Fig. 3 display the work hardening behaviors obtained when the same values of $n$ and $C_{1}$ are considered for all slip families. In this case, there is no strain hardening rate increase during stage B and no inversion of strain rate effect between RD and TD orientations. In the transition between stage A and $C$, the higher the strain rate the lower the $\Theta$ value for both orientations.

Fig. 4 illustrates the quality of prediction of the stress-strain curves (Fig. 4). It can be seen that the agreement is indeed very fine for TD samples. The strain rate effect is globally retrieved for both orientations although the beginning of RD curves displays too much work hardening. The latter result will receive explanation in Section 6.

\subsection{Slip activity}

The physics of the model is also assessed by checking the activity of different slip families and the Lankford coefficients. The relative activity of a slip family $(\alpha)$ can be given by

$$
\alpha=\frac{\sum_{g=1}^{n g} \sum_{s=p}^{q} f_{g}\left|\dot{\gamma}_{g}^{(s)}\right|}{\sum_{g=1}^{n g} \sum_{s=1}^{n s} f_{g}\left|\dot{\gamma}_{g}^{(s)}\right|}
$$

where $f_{g}$ is the grain volume fraction, $n g$ the total number of grains, $p$ and $q$ the first and the last slip system number of the family and $n s$ the total number of slip systems (Table 2). Fig. 5 shows the evolution of the activity of different slip families for two orientations. The activity of $P$ systems predominates at the very beginning of the deformation for both orientations, which is in agreement with some recent conclusions that $P$ slip may govern the $0.2 \%$ yield stress in RD and TD (Won et al., 2015). Nonetheless, it decreases much faster in TD orientation and stabilizes at a level comparable to $\Pi_{1}^{<a>}$ activity. In $R D$

Table 9

Model parameters that are not specific to slip families.

\begin{tabular}{lllllll}
\hline$v_{0}$ & $\rho_{m_{0}}$ & $\rho_{f_{0}}$ & $C_{2}$ & $K$ & $a_{\text {coli }}$ & $a_{c}$ \\
\hline $3 \cdot 10^{-5} \mathrm{~ms}^{-1}$ & $1 \cdot 10^{10} \mathrm{~m}^{-2}$ & $5 \cdot 10^{12} \mathrm{~m}^{-2}$ & 75 & 80 & 0.7 \\
\hline
\end{tabular}



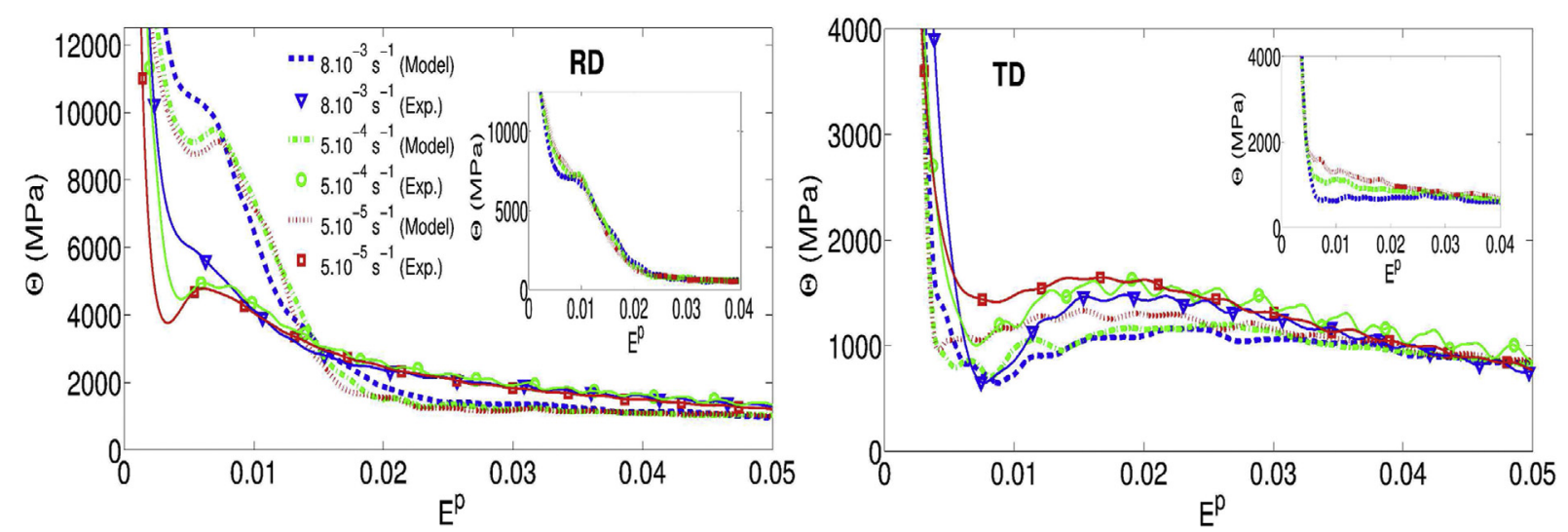

Fig. 3. Comparison of the work hardening rate evolution as a function of plastic strain between model predictions and experimental measurements. Inset: results of simulations with single values of $n$ and $C_{1}$ for all slip families $\left(n=50\right.$ and $\left.C_{1}=1.5 \cdot 10^{-4}\right)$.

orientation, $P$ activity predominates all over the strain range. It is characterized by a slow decrease in the first $2 \%$ of plastic strain and than by a roughly constant level. These predictions agree qualitatively with the frequencies of slip line families observed experimentally (Tables 4 and 5). Furthermore, it should be recalled that the experimental measurements do not provide such an integrate information on the slip activity for all slip systems within a grain, but only for the most active one. To provide a more quantitative comparison, a second frequency is computed from the model with the aim of mimicking the experimental procedure as follows:

- in each grain, only the system with the highest amount of $\operatorname{slip}\left(\gamma_{\max }^{(s)}\right)$ is considered,

- if this amount is greater than a critical value $\left(\gamma_{\max }^{(s)}>\gamma_{c}\right)$, the grain is considered to have observable slip lines,

- frequencies of slip families are then computed for the grains retained in the statistics, with one slip family per grain.

Fig. 5 presents such data. The main consequence of this second method of computation is an increase in the $P$ frequency. The agreement with the experimental points is then better. It can be noted that the model still predicts some $B$ activity whereas almost no basal slip line was observed in our specimens. According to the second method of frequency computation, their proportion remains nevertheless low $(<4 \%$ in $\mathrm{RD}$ and $<13 \%$ in TD) and consistent with other experimental data listed in the literature (Li et al., 2013; Barkia et al., 2015).

Figs. 5 and 6 show clearly that strain rate modifies the selection of active slip systems in the model. At low strains, $P$ activity increases with strain rate while that of $\Pi_{1}^{\langle a\rangle}$ decreases. This effect comes from the assumption of a higher $n$ value for $P$ systems (Table 8), which means that the stress required for $P$ slip grows slower with strain rate than for the other systems (Eq. (4)).

\subsection{Lankford coefficients}

Table 10 compares the measured and predicted Lankford coefficients. Literature experimental data for samples with similar composition loaded at $10^{-3} \mathrm{~s}^{-1}$ are also cited and show a good agreement with the present study (Benmhenni
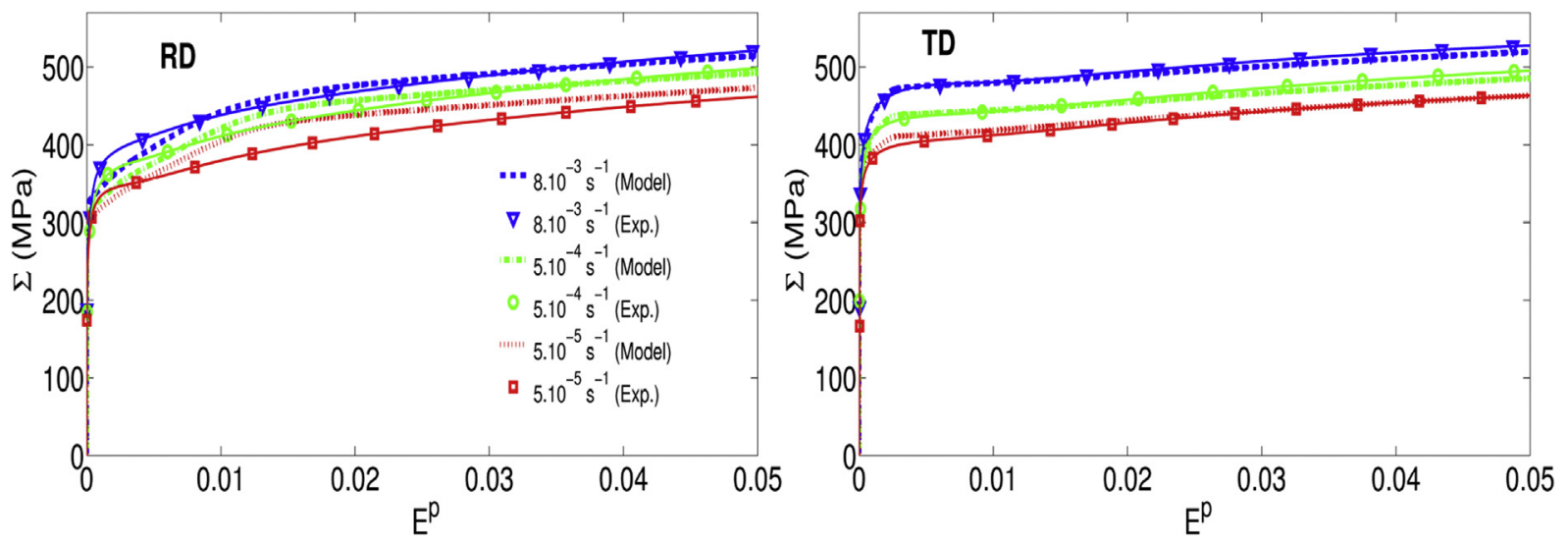

Fig. 4. Comparison of tensile stress-strain curves between model predictions and experimental measurements. 

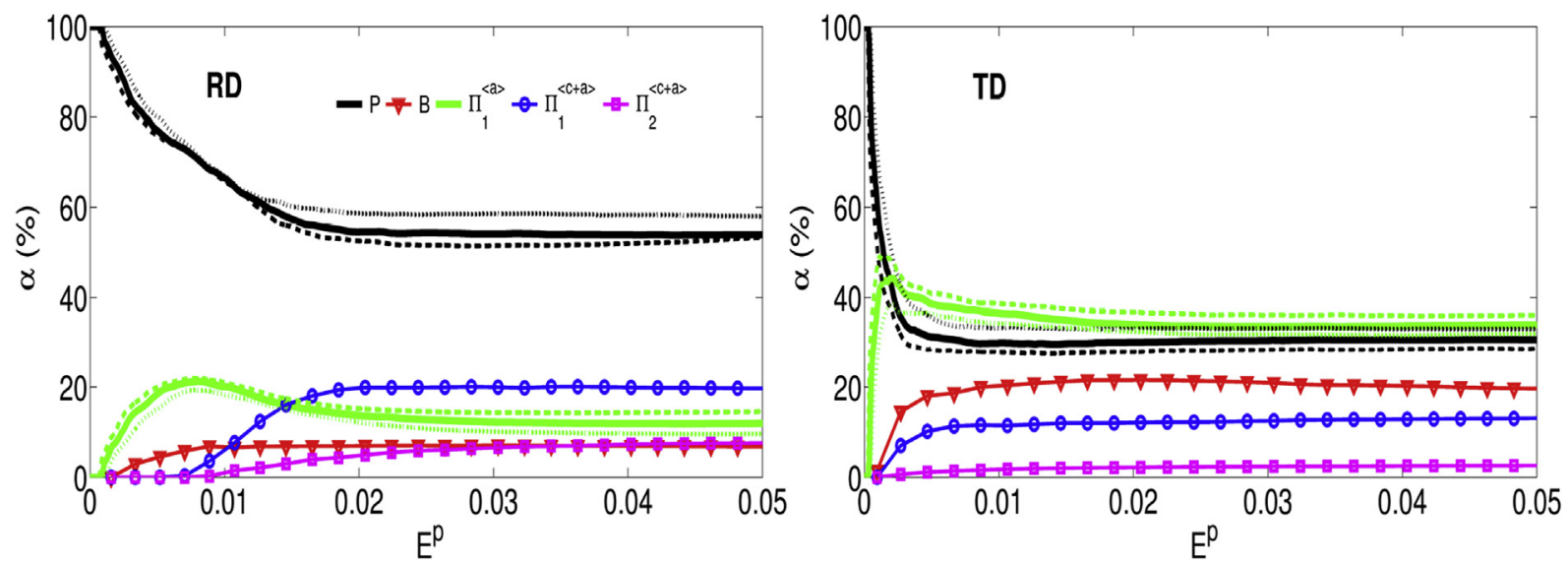

Fig. 5. Relative activities of slip families $(\alpha)$ predicted by the model. The strain rate effect on $P$ and $\Pi_{1}^{\langle a\rangle}$ families is shown: $5 \cdot 10^{-5} \mathrm{~s}^{-1}\left(\mathrm{dashed}\right.$ lines), $5 \cdot 10^{-4} \mathrm{~s}^{-1}$ (solid lines), $8 \cdot 10^{-3} \mathrm{~s}^{-1}$ (dotted lines).

et al., 2013). The evolution of the Lankford coefficients as predicted by the model is displayed in Fig. 7. While it is predicted that the Lankford coefficients of TD-samples follow an exponential-like decrease, the predicted coefficients of RD-samples are almost constant since the very beginning of plastic deformation. It can be observed that the strong strain anisotropy characterizing CP Ti is qualitatively retrieved by the model. From the quantitative point of view, the predicted Lankford coefficient matches well the experimental value for the RD orientation $(r \sim 2)$ but underestimates it for the TD orientation $(r \sim 4 / r \sim 5)$. Benmhenni et al. (2013) showed that the estimation of Lankford coefficients in CP Ti is very sensitive to the sharpness of the texture and slip systems activities. The absence of intragranular misorientations in the model and/or small difference compared to real slip systems activities (Fig. 6) may hence explain the observed discrepancy in TD orientation. Besides, the effect of anisotropic elasticity on the present results, which includes the use of directional shear moduli in the Taylor relation (Eq. (5)), should be underlined. The Lankford coefficients that are obtained within the framework of isotropic elasticity (Table 10) illustrate the fact that the repartition of slip systems activities is significantly modified compared with the anisotropic framework. In particular, the activity of $P$ systems decreases while it increases for $B$ systems.

\subsection{Textures evolution}

Figs. 8 and 9 compare the measured and predicted textures after deformation for RD and TD-samples, respectively. Since no obvious effect of the applied strain rate on textures evolution was observed, only the tests at $5 \cdot 10^{-4} \mathrm{~s}^{-1}$ are displayed. The simulations were stopped around $10 \%$ because of the use of a small strain framework. It can thus be noted that the predicted pole figures are given at lower strains $\left(E^{p}=0.11\right)$ than the measured ones $\left(E^{p}=0.18\right)$.
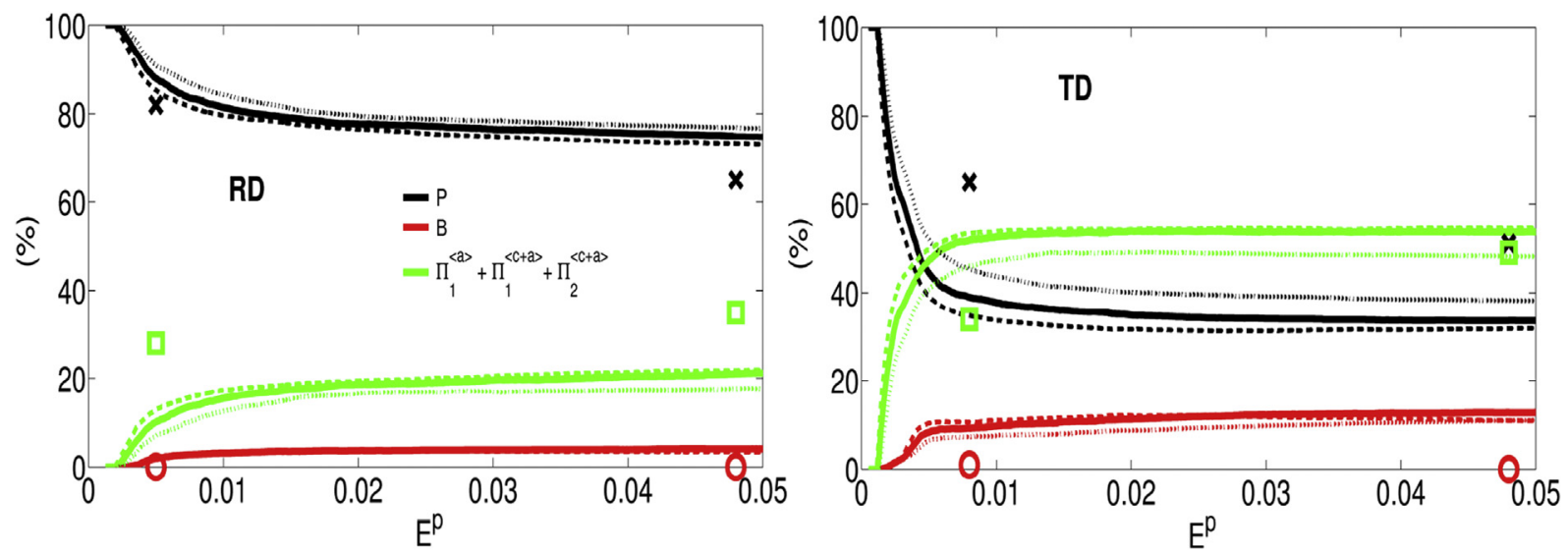

Fig. 6. Frequency of observable slip families as estimated by the model ( $\gamma_{c}=0.004$, see text). The strain rate effect is shown: $5 \cdot 10^{-5} \mathrm{~s}^{-1}$ (dashed lines), $5 \cdot 10^{-4} \mathrm{~s}^{-1}$ (solid lines), $8 \cdot 10^{-3} \mathrm{~s}^{-1}$ (dotted lines). Data points correspond to the experimental values of Tables 4 and 5 taken at $5 \cdot 10^{-4} \mathrm{~s}^{-1}$. The values measured at necking correspond in reality to larger strain $\left(E^{p}>0.05\right)$. 
Measured and predicted Lankford coefficients. "A" stands for the anisotropic elasticity case and "I" for the isotropic elasticity case. In the latter case, simulations are run with $\nu=\mathbf{0 . 3}$ and $\mu=42.25 \mathrm{GPa}$, the other parameters being the same as in the anisotropic case. ${ }^{*}$ The values of Benmhenni et al. (2013) are actually obtained thanks to a linear regression over a large strain interval.

\begin{tabular}{lllll}
\hline & \multicolumn{2}{l}{ Experimental measurements } & & Model predictions (A//I) \\
\cline { 2 - 3 } & $\begin{array}{l}5 \cdot 10^{-4} \mathrm{~s}^{-1} \\
\text { (this study) }\end{array}$ & $\begin{array}{l}10^{-3} \mathrm{~s}^{-1} \\
(\text { Benmhenni et al., 2013) }\end{array}$ & & $5 \cdot 10^{-5} \mathrm{~s}^{-1}$ \\
\hline $\mathrm{RD}\left(E^{p}=9.0 \%\right)$ & 2.1 & $2.2^{*}$ & $2.0 / / 1.8$ & $1.9 / / 1.7$ \\
$\operatorname{TD}\left(E^{p}=6.2 \%\right)$ & 5.5 & $4.9^{*}$ & $3.6 / / 3.1$ & $3.7 / / 3.2$ \\
\hline
\end{tabular}

There is obviously no marked change in the basal $\{0002\}$ pole figure of RD-samples (Fig. 8). The texture after tensile deformation along TD displayed at the top of Fig. 9 corresponds actually to the sample with the most important twin volume fraction (5.7\% T, see Table 3). The effect of twinning can then be seen at the top of Fig. 9, with an almost $90^{\circ}$ reorientation of some of the basal poles which were virtually aligned with the tensile axis. This twinning effect is nevertheless restricted to a small volume fraction and, similarly to RD-samples, the vast majority of TD sample basal poles keep their position. On the contrary, the two other pole figures show significant changes for both orientations. The prismatic $\{10 \overline{1} 0\}$ poles which were initially almost evenly distributed around the basal poles (Figs. 1 and 2) are now aligned with the tensile axis, i.e. along RD for RD-samples and along TD for TD-samples (Figs. 8 and 9). This feature is consistent with previous observations on similar materials (e.g., Becker and Pantleon (2013) for RD-samples). The alignement of the prismatic poles with the tensile axis is qualitatively very well retrieved by the model for both RD and TD-samples (see the bottom of Figs. 8 and 9). Hence, the comparison of predicted and measured textures corroborates the predictions of the evolution of the slip activity in different systems.

\subsection{Dislocation densities}

Fig. 10 exhibits the evolution of the total forest and total mobile dislocation densities. The densities are much higher in RD orientations since the fraction of plastic strain that is achieved by glide on $P$ systems is much more significant (Fig. 5). Indeed, the choice of a greater $C_{1}$ value for $P$ systems (Table 8 ) implies that the corresponding density of mobile but also of forest dislocations grows faster than for the other systems (Eqs. (6) and (7)). As a result, the contribution of $P$ systems to the global strain hardening rate is preponderant because of a faster decrease of the dislocations mean free path (Eq. (8)). This scenario may explain the stronger work hardening rate for RD than for TD during stage C (cf. Roth et al. (2014) and Fig. 3), in spite of a less significant twinning and a higher frequency of slip on easy systems. On the contrary, simulations that are run with a single value of $C_{1}$ for all slip families display higher dislocation densities in TD-samples (insets of Fig. 10) and give stronger hardening during stage $C$ for traction along TD direction (not shown).

Besides, Fig. 10 shows that the higher the strain rate the higher the dislocation density, even when simulations are run with same values of $n$ and $C_{1}$ for all slip families. This effect is thus intrinsically related to Eqs. (6) and (7). Of course, it is intensified by the increase of $P$ activity with strain rate since the production of mobile dislocations is stronger on $P$ systems.

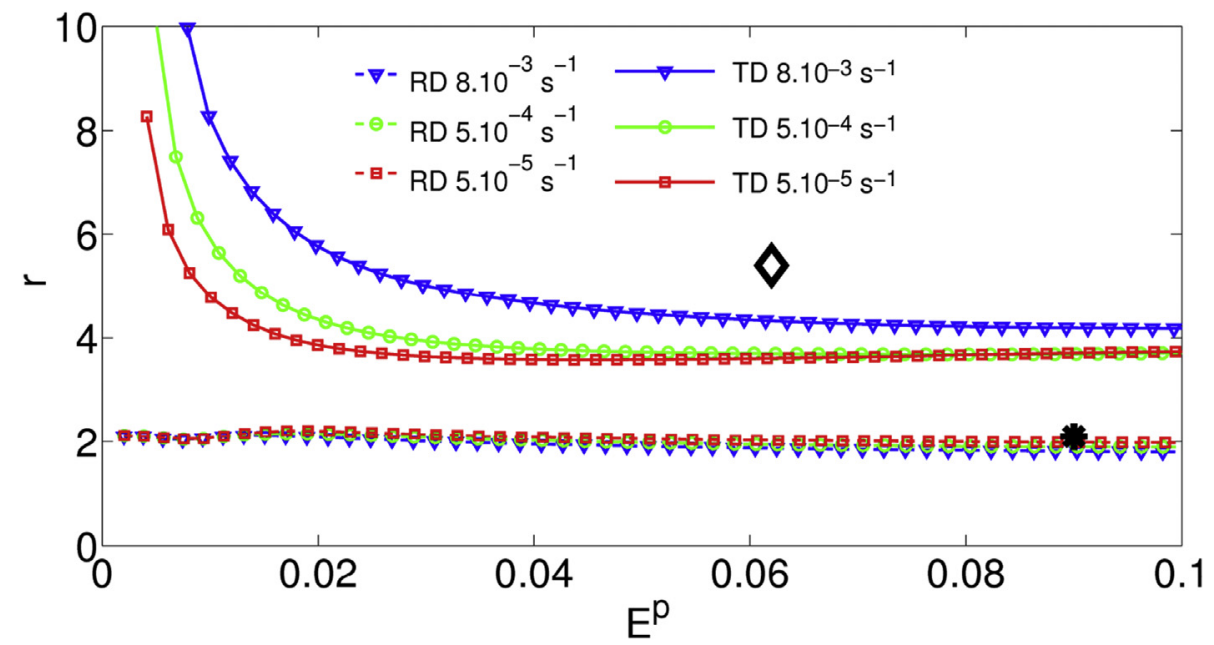

Fig. 7. Evolution of the Lankford coefficients as predicted by the model. Data points corresponds to experimental measurements at $5 \cdot 10^{-4} \mathrm{~s}^{-1}$ (RD: star symbol; TD: diamond symbol). 

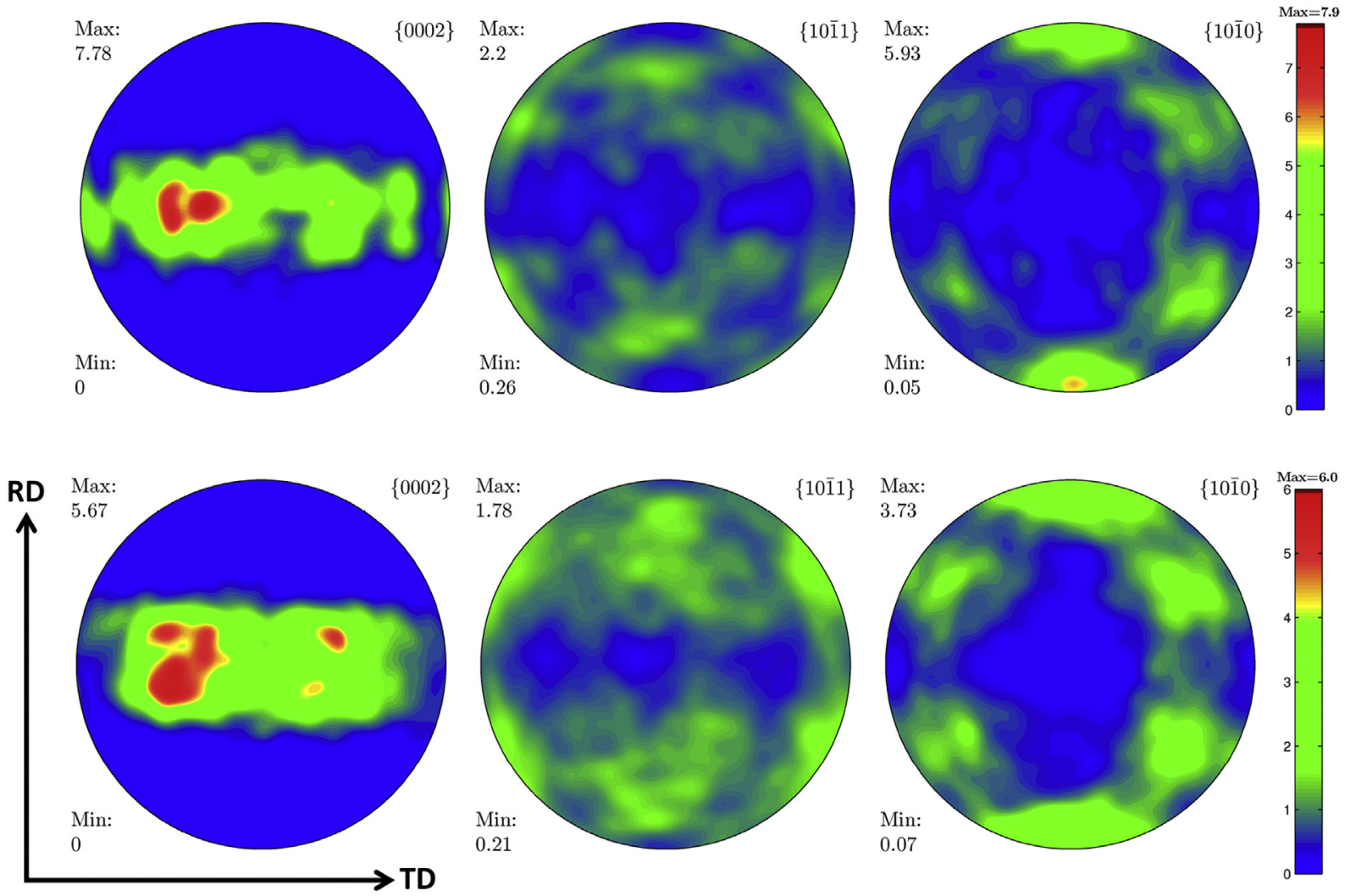

Fig. 8. Comparison between measured (top, $E^{p}=0.18$ ) and predicted (bottom, $E^{p}=0.11$ ) pole figures for RD samples deformed at $5 \cdot 10^{-4} \mathrm{~s}^{-1}$. The plots were performed with the MTEX toolbox (Bachmann et al., 2010).

\subsection{Plastification of the grains}

To understand the initial work hardening behavior, it is also interesting to see how the progressive plastification of the grains takes place in the model. Fig. 11 shows the evolution of the proportion of grains with either one or two activated system. It is seen that plastic yielding occurs in a more diffuse way in TD-samples than in RD-samples. Moreover, in TDsamples, the secondary slip starts sooner and the number of grains with two activated systems grows quicker. This observation is consistent with higher experimental values of the yield stress in the case of TD-samples (Roth et al., 2014; Won et al., 2015). At the same time, no clear effect of strain rate on plastic yielding can be noticed from Fig. 11.

\section{Discussion}

It should be underlined that all the equations of the present model produce monotonic responses but still allow to achieve a non monotonic behavior of $\Theta$. Theoretically, the introduction of mobile dislocations by itself may produce a non monotonic behavior of $\Theta$, since the increase of the latter induces softening while the increase of forest dislocations induces hardening. However, insets in Fig. 3, which display the work hardening behaviors obtained with mobile dislocation density evolution but when the same values of $n$ and $C_{1}$ are considered for all slip families, show that no strain hardening rate increase during stage $B$ is obtained. Another contribution to the non monotonicity of $\Theta$ thus arises from the complex interplay between slip family activities. Indeed, in the present model, each slip family self-hardens and hardens the other systems differently, due to specific shear moduli, interactions coefficients, forest dislocation density evolutions and strain rate sensitivities. For instance, the activation of $\Pi_{1}^{<a>}$ slip induces strong hardening as a result of collinear interactions with $P$ systems (see Eq. (5)) which have already been sheared significantly and thus hold high dislocations densities due notably to a high $C_{1}$ value. Indeed, in RD-samples, the $\Theta$ increase during stage B correlates with the increase in $\Pi_{1}^{\langle a\rangle}$ activity (Figs. 3 and 5), as well as the growing importance of secondary slip (Fig. 11). The value of the collinear interaction coefficient has thus a significant influence on the $\Theta$ increase. In TD-samples, stage B is also characterized by a growing importance of secondary slip. This effect is however less obvious since more than $65 \%$ of grains are already performing multiple slip at the end of stage $A$.

In the model, the wells in the $\Theta$ evolution happen around $0.5 \%$ and $0.8 \%$ of plastic strain for RD and TD-samples, respectively (Fig. 3). These strains correspond precisely to the instants at which virtually all grains are plastified (Fig. 11). 


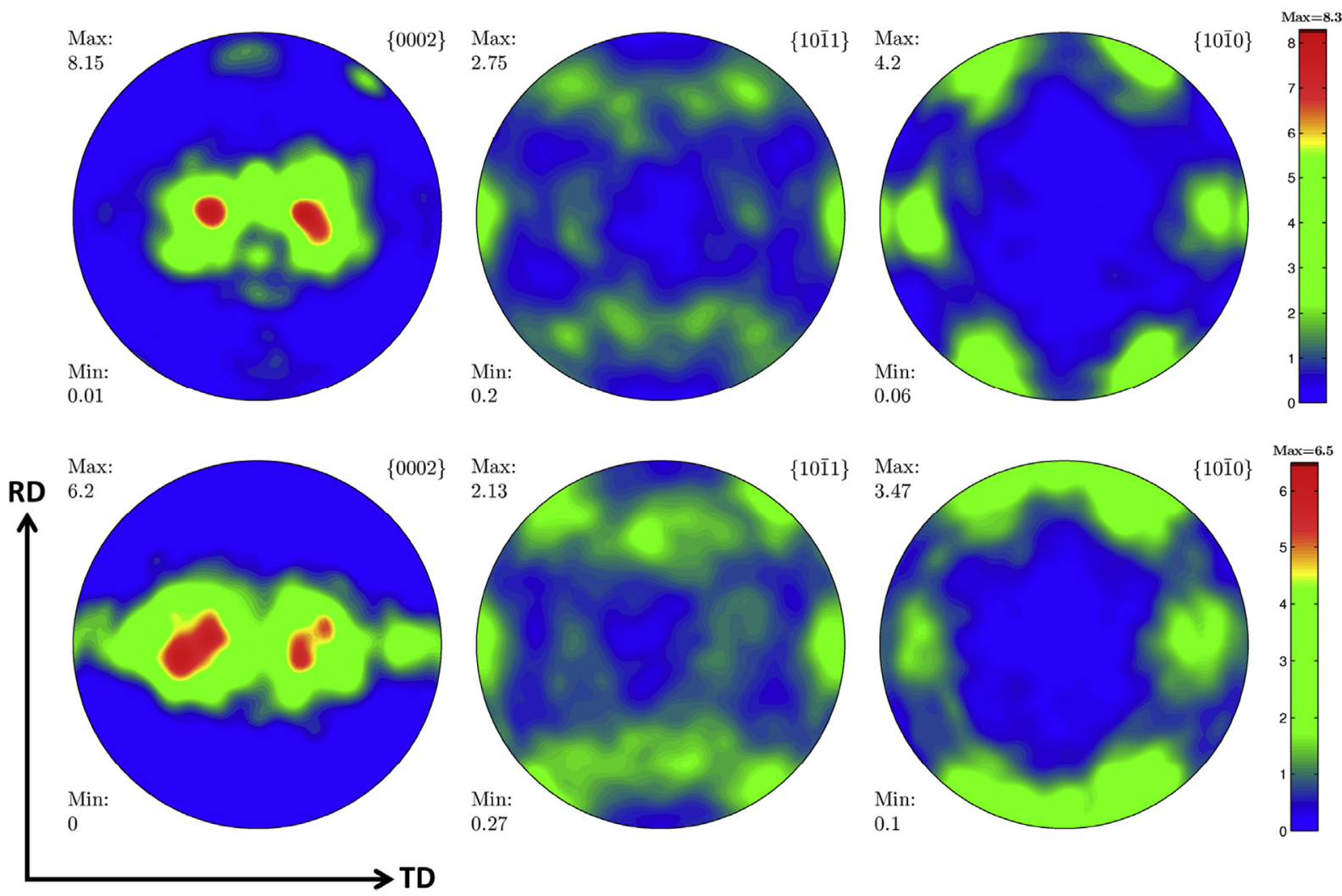

Fig. 9. Comparison between measured (top, $E^{p}=0.18$ ) and predicted (bottom, $E^{p}=0.11$ ) pole figures for TD samples deformed at $5 \cdot 10^{-4} \mathrm{~s}^{-1}$. The plots were performed with the MTEX toolbox (Bachmann et al., 2010).

As a consequence, the $\Theta$ decrease during stage A should be related to the progressive plastic yielding of the grains, as also conjectured by Barkia et al. (2015). For RD-samples, it can be deduced from Figs. 5 and 11 that most grains deform by single slip on a $P$ system during stage A. For TD-samples, the situation is more complex with more than $65 \%$ of grains performing multiple slip at the end of stage A (Fig. 11) and a comparable amount of $P$ and $\Pi_{1}^{<a>}$ slip (Fig. 5). In particular, plastic yielding is much more gradual in TD-samples, which certainly explains why they first display a stronger strain hardening rate than RDsamples at the very beginning of plastic deformation (not shown) and then, a lower strain hardening rate towards the end of stage A (Fig. 3).
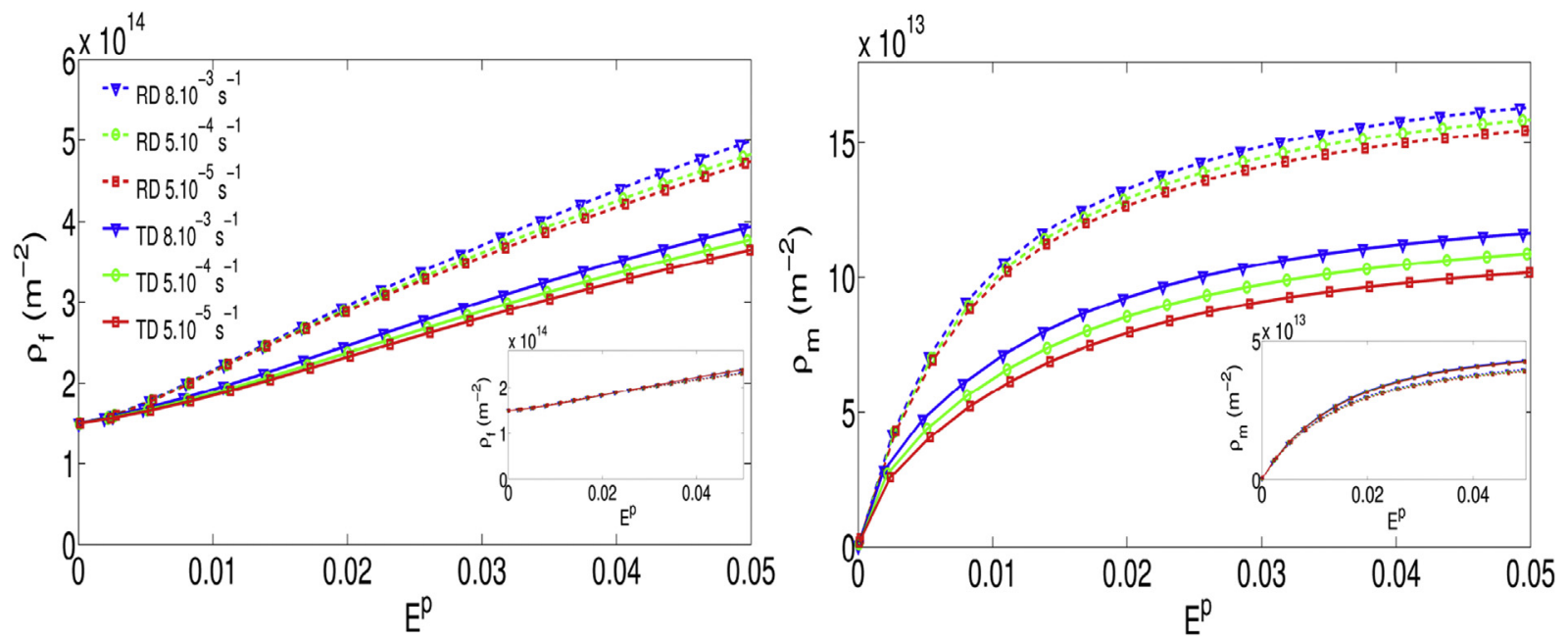

Fig. 10. Model predictions for the evolution of the total forest $\left(\rho_{f}\right)$ and mobile $\left(\rho_{m}\right)$ dislocation densities (i.e., densities are cumulated over the 30 slip systems and averaged over the grains population). Inset: results of simulations with single values of $n$ and $C_{1}$ for all slip families $\left(n=50\right.$ and $\left.C_{1}=1.5 \cdot 10^{-4}\right)$. 


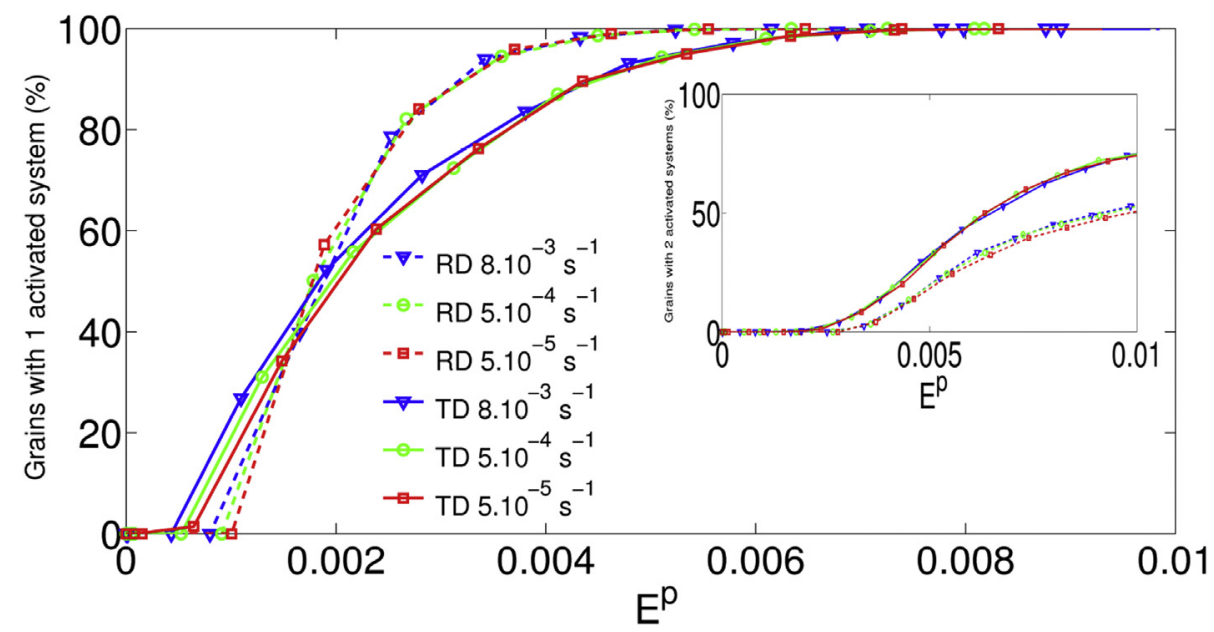

Fig. 11. Proportion of grains with one activated system (i.e., grains with $\gamma>0.004$ on the most active system). Inset: proportion of grains with two activated systems (i.e., grains with $\gamma>0.004$ on the second most active system).

Simulations ran with single values of $n$ and $C_{1}$ for all slip families retrieve qualitatively the strain rate effect on the work hardening behavior during stage A in TD-samples (Fig. 3). This result indicates that, in TD-samples, the well depth of the strain hardening rate should be first and foremost influenced by the production of mobile dislocations. The latter is indeed all the more pronounced as strain rate increases, whatever the assumptions about $n$ and $C_{1}$. On the contrary, concerning RD-samples, the assumption of a specific SRSS for $P$ slip is essential to retrieve the effect of strain rate. In this case, the work hardening behavior during stage A should be first and foremost influenced by the selection of active slip systems. From Fig. 5, it is established that, at low strains, the proportion of $P$ activity increases with strain rate. At first glance, one could conjecture that the enhancing $P$ slip should forcedly lead to a lower work hardening rate since the production of mobile dislocations is assumed easier on $P$ systems. However, since the $P$ family has the lowest CRSS, the activation of a $P$ system instead of another one also means that slip takes place on a less well-oriented system corresponding to a lower component $R_{l l}$ of the Schmid orientation tensor, $l$ being the loading direction (cf. Eq. (2) and the expression of the resolved shear stress in Section 4.1). As a consequence, the slip contribution to the specimen axial elongation is weaker (Eq. (2)) and may induce a stronger strain hardening rate. Fig. 12 illustrating this effect displays the evolution of the parameter $\beta$ which stands for the average contribution of slip on the most active system to the specimen axial elongation. This parameter is defined as follows

$$
\beta=\sum_{g} f_{g}\left|R_{l l}^{(a)}\right|
$$

where $l$ designates the loading direction and ( $a$ ) the most active system in grain ( $g$ ). Fig. 12 shows indeed that the parameter $\beta$ decreases with the strain rate.

Thus, the model is able to explain qualitatively all specific experimental observations. While one should not seek for a perfect quantitatve agreement, it is noteworthy that the overestimation of the work hardening rate during stage A in RDsamples seems to have a natural explanation. Indeed, since almost all grains deform by single slip during this stage, plastic accommodation should be difficult and sizeable inter-granular stresses are expected. It has been demonstrated that affine formulations provide better predictions of internal stresses than secant ones (Mareau and Berbenni, 2015). However, 1-site self-consistent models are known to overestimate internal stresses compared to full field methods which do allow for the neigboring effects (Mareau and Berbenni, 2015). This statement is particularly true in the case of a strong non-linearity, such as suggested in the present work $(n=32$ and $n=65)$ to account quantitatively for strain rate effects.

\section{Conclusions}

An elasto-viscoplastic self-consistent model, based on the translated field method for an affine linearization of the viscoplastic flow rule recently introduced by Mareau and Berbenni (2015), was developed to investigate the three-stage work hardening behavior of CP Ti observed in tensile condition by Roth et al. (2014): a strong decrease of strain hardening rate during stage A followed by an increase in stage B and a new decrease in stage $C$. In particular, the experiments revealed an opposite effect of the strain rate on the well depth, at the transition between stages A and B, depending on whether specimens were elongated along the rolling or the transverse direction (Roth et al., 2014). The microstructure analysis realized in the present work provided a physical basis for modeling such peculiar behavior in tensile condition. First, interrupted tensile tests 


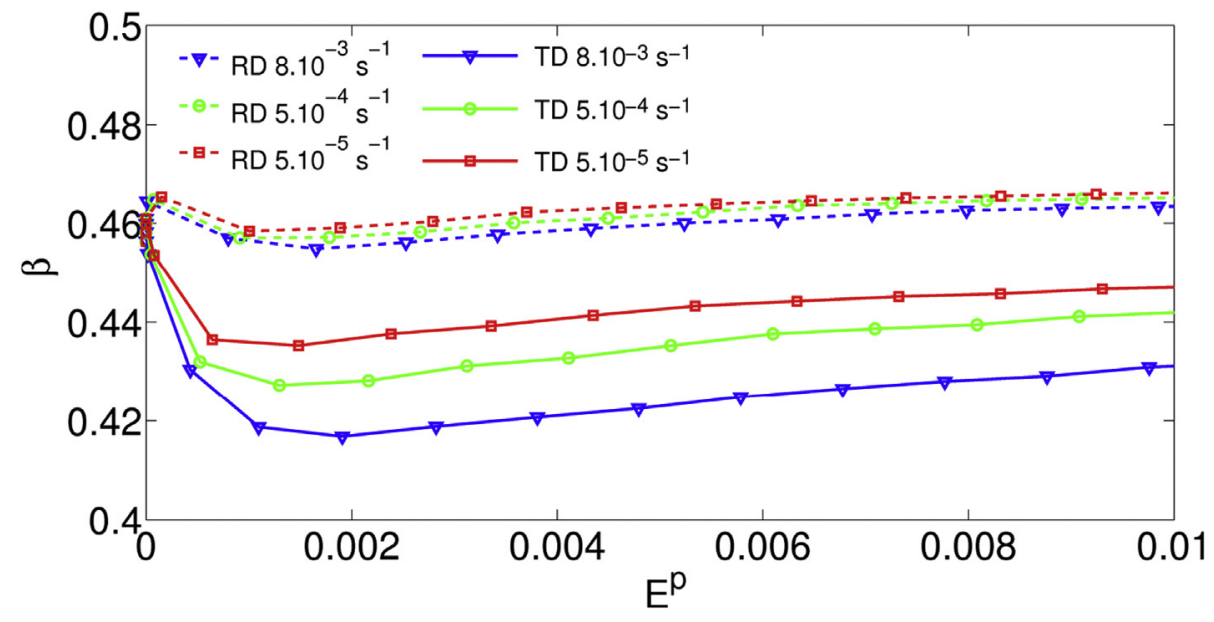

Fig. 12. Average contribution of slip on the most active system to the specimen axial elongation ( $\beta$, see Eq. (22)).

confirmed that the volume fraction of twins was too small around stage B to ascribe the increase of strain hardening rate to twins. Then, the slip lines analysis revealed a strong initial predominance of prismatic slip, particularly pronounced in specimens strained along the rolling direction. The relative activity of primsatic slip was observed to decrease with the samples deformation and to increase with the applied strain rate. According to these different observations, it was suggested that the three-stage hardening character could be explained using mechanisms based on dislocation glide only, in particular by assuming a lower SRSS for prismatic systems.

Hence, the model considered crystal plasticity framework. It distinguished between mobile and forest dislocations in order to simulate fast initial multiplication of the mobile dislocations. This feature is consistent with the arguments proposed by Conrad (1981) and Naka (1983) to explain the presence of a yield plateau in Ti: the rapid multiplication of dislocations induces a drop in their average glide velocity, so that the increase in the flow stress due to hardening is considerably reduced. The model also assumed a higher dislocation multiplication rate for prismatic systems in order to relate the higher work hardening rate observed along $\mathrm{RD}$ during stage $\mathrm{C}$ to a higher dislocation density. Furthermore, it should be underlined that the model adopted the framework of anisotropic elasticity, which includes the use of directional shear moduli in the Taylor relation (Eq. (5)), and acknowledged stronger interactions between collinear slip systems in accordance with DDD simulations (Madec et al., 2003; Hoc et al., 2004; Devincre et al., 2005, 2006; Kubin et al., 2008; Queyreau et al., 2009; Devincre, 2013; Bertin et al., 2014). These two features allowed for taking into account, at least partially, the strong anisotropy of the CRSSs, by virtue of physically-based parameters. Based on these different assumptions, stress-strain curves were correctly reproduced and sound estimates of Lankford coefficient, prismatic slip activity and textures evolution were obtained. Most importantly, the simulations were able to explain the opposite effect of strain rate with regard to the orientation of the tensile axis. They revealed two antagonist effects of strain rate on the work hardening behavior. On the one hand, the increasing strain rate promotes the selection of easy prismatic glide among available slip systems. As a consequence, the slip contribution to the specimen axial elongation is weaker, which may actually induce a stronger strain hardening rate. This effect is conjectured to be predominant in RD-specimens. On the other hand, the production of mobile dislocations increases with strain rate and, consequently, enlarges the well depth at the end of stage A. This effect is conjectured to be predominant in TDspecimens.

The results obtained may be of interest in a larger context concerning modeling of plasticity of Ti or, more generally, hexagonal materials. Further progress may come from considering both dislocation glide and twinning, which should allow applying the model to other loading modes. Having access to more accurate estimates of the interaction coefficients in Ti would also help improving the model predictive capacity. Finally, it would be interesting to see the grain size distribution effect on the present results by accounting for a grain size effect in the computation of the CRSSs (Eq. (5)) and/or in the computation of the dislocation mean free paths (Eq. (8)) as done theoretically by Berbenni et al. (2007) and applied in titanium by Fromm et al. (2009) and in steels by Nicaise et al. (2011).

\section{Acknowledgments}

The authors gratefully acknowledge Stéphane Berbenni for very valuable advice on the affine elasto-viscoplastic selfconsistent modeling, Jean-Sébastien Lecomte for his help with texture characterization and Lu Tuan Le for fruitful discussion of the work hardening modeling. This work was supported by the French State through the program "Investment in the future" operated by the National Research Agency (ANR) and referenced by ANR-11-LABX-0008-01 (LabEx DAMAS). 


\section{References}

Bachmann, F., Hielscher, R., Schaeben, H., 2010. Texture analysis with mtex - free and open source software toolbox. Sol. State Phenom. 160, 63-68.

Barkia, B., Doquet, V., Couzinié, J.P., Guillot, I., Héripré, E., 2015. In situ monitoring of the deformation mechanisms in titanium with different oxygen contents. Mat. Sci. Eng. A 636, 91-102.

Basinski, Z.S., Szcerba, M.S., Niewczas, M., Embury, J.D., Basinski, S.J., 1997. The transformation of slip dislocations during twinning of copper-aluminium alloy crystals. Rev. Metall. 94, 1037-1043.

Becker, H., Pantleon, W., 2013. Work-hardening stages and deformation mechanism maps during tensile deformation of commercially pure titanium. Comput. Mat. Sci. 76, 52-59.

Benmhenni, N., Bouvier, S., Brenner, R., Chauveau, T., Bacroix, B., 2013. Micromechanical modelling of monotonic loading of cp $\alpha$-ti: correlation between macroscopic and microscopic behaviour. Mat. Sci. Eng. A 573, 222-233.

Berbenni, S., Favier, V., Berveiller, M., 2007. Impact of the grain size distribution on the yield stress of heterogeneous materials. Int. J. Plast. 23, 114-142.

Bertin, N., Tomé, C.N., Beyerlein, I., Barnett, M.R., Capolungo, L., 2014. On the strength of dislocation interactions and their effect on latent hardening in pure magnesium. Int. J. Plast. 62, 72-92.

Chichili, D.R., Ramesh, K.T., Hemker, K.J., 1998. The high-strain-rate response of alpha-titanium: experiments, deformation mechanisms and modeling. Acta Mater 46, 1025-1043.

Churchman, A.T., 1954. The slip modes of titanium and the effect of purity on their occurence during tensile deformation of single crystals. Proc. R. Soc. A 226, 216-226.

Conrad, H., 1981. Effect of interstitial solutes on the strength and ductility of titanium. Prog. Mat. Sci. 26, 123-403.

Devincre, B., 2013. Dislocation dynamics simulations of slip systems interactions and forest strengthening in ice single crystal. PhiPhilos.l. Mag. 93, 235-246.

Devincre, B., Hoc, T., Kubin, L.P., 2005. Collinear interactions of dislocations and slip systems. Mat. Sci. Eng. A 400-401, 182-185.

Devincre, B., Kubin, L., Hoc, T., 2006. Physical analyses of crystal plasticity by dd simulations. Scr. Mater 54, 741-746.

Doner, M., Conrad, H., 1973. Deformation mechanisms in commercial ti-50a (0.5 at. pct o)eq at intermediate and high temperatures (0.3-0.6 t)m. Metall. Trans. 4, 2809-2817.

Estrin, Y., Kubin, L.P., 1986. Local strain hardening and nonuniformity of plastic deformation. Acta Metall. 34, 2455-2466.

Franciosi, P., Berveiller, M., Zaoui, A., 1980. Latent hardening in copper and aluminium single crystals. Acta Metall. 28, $273-283$.

Fromm, B.S., Adams, B.L., Ahmadi, S., Knezevic, M., 2009. Grain size and orientation distributions: application to yielding of $\alpha$-titanium. Acta Mater 57, $2339-2348$.

Ghaderi, A., Barnett, M.R., 2011. Sensitivity of deformation twinning to grain size in titanium and magnesium. Acta Mater 59, 7824-7839.

Gloagen, D., Oum, G., Legrand, V., Fajoui, J., Branchu, S., 2013. Micromechanical modelling of monotonic loading of cp $\alpha$-ti: correlation between macroscopic and microscopic behaviour. Acta Mater 61, 5779-5790.

Gong, J., Wilkinson, A.J., 2009. Anisotropy in the plastic flow properties of single-crystal $\alpha$-titanium determined from micro-cantilever beams. Acta Mater 57, 5693-5705.

Gurao, N.P., Kapoor, R., Suwas, S., 2011. Deformation behaviour of commercially pure titanium at extreme strain rates. Acta Mater 59, 3431-3446.

Hearmon, R.F., 1979. The elastic constants of crystals and other anisotropic materials. In: Hellwege, K.-H., Hellwege, A.M. (Eds.), Landolt-Börnstein Tables, Group III, vol. 11. Springer-Verlag, Berlin, 1-154.

Hoc, T., Devincre, B., Kubin, L., 2004. Deformation stage i of fcc crystals: constitutive modelling. In: Gundlach, C.E.A. (Ed.), Evolution of Deformation Microstructures in 3D. Risoe National Laboratory, Roskilde, Denmark, pp. 43-59.

Knezevic, M., Lebensohn, R.A., Cazacu, O., Revil-Baudard, B., Proust, G., Vogel, S.C., Nixon, M.E., 2013. Modeling bending of $\alpha$-titanium with embedded polycrystal plasticity in implicit finite elements. Mat. Sci. Eng. A 564, 116-126.

Kröner, E., 1989. Modified green functions in the theory of heterogeneous and/or anisotropic linearly elastic media. In: Weng, G.J., Taya, M., Abe, M. (Eds.), Micromechanics and Inhomogeneity. Springer, Berlin, pp. 197-211.

Kubin, L., Devincre, B., Hoc, T., 2008. Modeling dislocation storage rates and mean free paths in face-centered cubic crystals. Acta Mater 56, 6040-6049.

Lecomte, J.S., Philipe, M.J., Klimanek, P., 1997. Plastic deformation of a ti-6\% al-4\% v alloy with a strong transverse-type crystallographic $\alpha$-texture at elevated temperatures. Mat. Sci. Eng. A 234-236, 869-872.

Levine, E.D., 1966. Deformation mechanisms in titanium at low temperatures. Trans. Metall. Soc. AIME 236, 1558-1565.

Li, H., Boehler, C.J., Bieler, T.R., Crimp, M.A., 2014. Analysis of the deformation behavior in tension and tension-creep of ti-3al-2.5v (wt pct) at $296 \mathrm{k}$ and $728 \mathrm{k}$ $\left(23^{\circ} \mathrm{C}\right.$ and $\left.455^{\circ} \mathrm{C}\right)$ using in situ sem experiments. Metall. Mat. Trans. A 45A, 6053-6066.

Li, H., Mason, D.E., Bieler, T.R., Boehler, C.J., Crimp, M.A., 2013. Methodology for estimating the critical resolved shear stress ratios of $\alpha$-phase ti using ebsdbased trace analysis. Acta Mater 61, 7555-7567.

Madec, R., Devincre, B., Kubin, L., Hoc, T., Rodney, D., 2003. The role of collinear interaction in dislocation-induced hardening. Science 301, $1879-1882$.

Mareau, C., Berbenni, S., 2015. An affine formulation for the self-consistent modeling of elasto-viscoplastic heterogeneous materials based on the translated fields method. Int. J. Plast. 64, 134-150.

Masson, R., Zaoui, A., 1999. Self-consistent estimates for the rate-dependentelastoplastic behaviour of polycrystalline materials. J. Mech. Phys. Sol. 47, $1543-1568$.

Minonishi, Y., Morozumi, S., Yoshinaga, H., 1982. $\{11 \overline{2} 2\}\langle 11 \overline{2} 3\rangle$ slip in titanium. Scr. Metall. 16, 427-430.

Monteiro, S.N., Reed-Hill, R.E., 1973. An empirical analysis stress-strain curves of titanium. Metall. Trans. 4, 1011-1015.

Naka, S., 1983. Etude des mécanismes de déformation plastique à basse température de monocristaux de titane $\alpha$ (Ph.D. thesis).

Naka, S., Lasalmonie, A., 1983. Cross-slip on the first order pyramidal plane (1011) of a-type dislocations [1210] in the plastic deformation of $\alpha$-titanium single crystals. J. Mat. Sci. 18, 2613-2617.

Nemat-Nasser, S., Guo, W.G., Cheng, J.Y., 1999. Mechanical properties and deformation mechanisms of a commercially pure titanium. Acta Mater 47, $3705-3720$.

Nicaise, N., Berbenni, S., Wagner, F., Berveiller, M., Lemoine, X., 2011. Coupled effects of grain size distributions and crystallographic textures on the plastic behaviour of if steels. Int. J. Plast. 27, 232-249.

Nixon, M.E., Cazacu, O., Lebensohn, R.A., 2010. Anisotropic response of high-purity $\alpha$-titanium: experimental characterization and constitutive modeling. Int. J. Plast. 26, 516-532.

Paquin, A., Berbenni, S., Favier, V., Lemoine, X., Berveiller, M., 2001. Micromechanical modeling of the elastic-viscoplastic behaviour of polycrystalline steels. Int. J. Plast. 17, 1267-1302.

Paquin, A., Sabar, H., Berveiller, M., 1999. Integral formulation and selfconsistent modelling of elastoviscoplastic behavior of heterogeneous materials. Arch. Appl. Mech. 69, 14-35.

Philippe, M.J., Serghat, M., Houtte, P.V., Esling, C., 1995. Modelling of texture evolution for materials of hexagonal symmetry ii: application to zirconium and titanium $\alpha$ or near $\alpha$ alloys. Acta Metall. Mater 43, 1619-1630.

Poty, A., Raulot, J.M., Xu, H., Schuman, C., Lecomte, J.S., Philippe, M.J., Esling, C., 2011. Classification of the critical resolved shear stress in the hexagonalclose-packed materials by atomic simulation: application to $\alpha$-zirconium and $\alpha$-titanium. J. Appl. Phys. 110, 014905(115).

Queyreau, S., Monnet, G., Devincre, B., 2009. Slip systems interactions in $\alpha$-iron determined by dislocation dynamics simulations. Int. J. Plast. $25,361-377$.

Reed-Hill, R.E., Iswaran, C.V., Kaufman, M.J., 1995. A power law model for the flow stress and strain-rate sensitivity in cp titanium. Scr. Metall. Mater 33, 157-162.

Roth, A., Lebyodkin, M.A., Lebedkina, T.A., Lecomte, J.S., Richeton, T., Amouzou, K.E.K., 2014. Mechanisms of anisotropy of mechanical properties of $\alpha$-titanium in tension conditions. Mat. Sci. Eng. A 596, 236-243. 
Salem, A.A., Kalidindi, S.R., Doherty, R.G., 2002. Strain hardening regimes and microstructure evolution during large strain compression of high purity titanium. Scr. Mater 46, 419-423.

Salem, A.A., Kalidindi, S.R., Doherty, R.G., 2003. Strain hardening of titanium: role of deformation twinning. Acta Mater 51, 4225 -4237.

Salem, A.A., Kalidindi, S.R., Doherty, R.G., Semiatin, S.L., 2006. Strain hardening due to deformation twinning in $\alpha$-titanium: mechanisms. Metall. Mat. Trans. A $37,259-268$.

Salem, A.A., Kalidindi, S.R., Semiatin, S.L., 2005. Strain hardening due to deformation twinning in $\alpha$-titanium : constitutive relations and crystal-plasticity modeling. Acta Mater 53, 3495-3502.

Wang, L., Wang, Y., Eisenlohr, P., Bieler, T.R., Crimp, M.A., Mason, D.E., 2010. Twin nucleation by slip transfer across grain boundaries in commercial purity titanium. Metall. Mat. Trans. A 41, 421-430.

Wang, S., Schuman, C., Bao, L., Lecomte, J.S., Zhang, Y., Raulot, J.M., Philippe, M.J., Zhao, X., Esling, C., 2012. Variant selection criterion for twin variants in titanium alloys deformed by rolling. Acta Mater 60, 3912-3919.

Wang, Y.N., Huang, J.C., 2003. Texture analysis in hexagonal materials. Mat. Chem. Phys. 81, 11-26.

Warwick, J.L.W., Jones, N.G., Rahman, K.M., Dye, D., 2012. Lattice strain evolution during tensile and compressive loading of cp ti. Acta Mater 60, 6720-6731.

Won, J.W., Park, K.T., Hong, S.G., Lee, C.S., 2015. Anisotropic yielding behavior of rolling textured high purity titanium. Mat. Sci. Eng. A 637, $215-221$.

Wu, X., Kalidindi, S., Necker, C., Salem, A.A., 2007. Prediction of crystallographic texture evolution and anisotropic stress-strain curves during large plastic strains in high purity $\alpha$-titanium using a taylor-type crystal plasticity model. Acta Mater 55, 423-432.

Xiaoli, T., Haicheng, G., Shufen, Z., Laird, C., 1994. Loading mode dependence of deformation microstructure in a high-purity titanium single crystal oriented for difficult glide. Mat. Sci. Eng. A 189, 77-84.

Zaefferer, S., 2003. A study of active deformation systems in titanium alloys : dependence on alloy composition and correlation with deformation texture. Mat. Sci. Eng. A 344, 20-30.

Zambaldi, C., Yang, Y., Bieler, T.R., Raabe, D., 2012. Orientation informed nanoindentation of a-titanium: indentation pileup in hexagonal metals deforming by prismatic slip. J. Mat. Res. 27, 356-367. 